\title{
Mouse Navigation Strategies for Odor Source Localization
}

\author{
Annie Liu' ${ }^{1,2+}$, Andrew E. Papale ${ }^{1 * t}$, James Hengenius ${ }^{3 t}$, Khusbu Patel ${ }^{4}$, \\ Bard Ermentrout ${ }^{2,3}$ and Nathan N. Urban ${ }^{1,2}$ \\ ${ }^{1}$ Department of Neurobiology, University of Pittsburgh School of Medicine, Pittsburgh, PA, United States, ${ }^{2}$ Center \\ for the Neural Basis of Cognition, Pittsburgh, PA, United States, ${ }^{3}$ Department of Mathematics, University of Pittsburgh, \\ Pittsburgh, PA, United States, ${ }^{4}$ Department of Neurobiology, University of Pittsburgh, Pittsburgh, PA, United States
}

OPEN ACCESS

Edited by:

Paul E. M. Phillips,

University of Washington,

United States

Reviewed by:

Daniel W. Wesson,

University of Florida, United States

David Henry Gire,

University of Washington,

United States

*Correspondence:

Andrew E. Papale

papalea@pitt.edu

†These authors have contributed equally to this work

Specialty section:

This article was submitted to

Decision Neuroscience,

a section of the journal

Frontiers in Neuroscience

Received: 23 December 2019

Accepted: 27 February 2020

Published: 20 March 2020

Citation:

Liu A, Papale AE, Hengenius J, Patel K, Ermentrout $B$ and Urban NN (2020) Mouse Navigation Strategies for Odor Source Localization.

Front. Neurosci. 14:218.

doi: 10.3389/fnins.2020.00218
Navigating an odor landscape is a critical behavior for the survival of many species, including mice. An ethologically relevant mouse behavior is locating food using information about odor concentration. To approximate this behavior, we used an open field odor-based spot-finding task indoors with little wind, examining navigation strategies as mice search for and approach an odor source. After mice were trained to navigate to odor sources paired with food reward, we observed behavioral changes consistent with localization $10-45 \mathrm{~cm}$ away from the source. These behaviors included orientation toward the source, decreased velocity, and increased exploration time. We also found that the amplitude of 'casting,' which we define as lateral back and forth movement of the nose, increased with proximity to the source. Based on these observations, we created a concentration-sensitive agent-based model to simulate mouse behavior. This model provided evidence for a binaral-sniffing strategy (inter-nostril comparison of concentration in a single sniff) and a serial-sniffing strategy (sampling concentration, moving in space, and then sampling again). Serial-sniffing may be accomplished at farther distances by moving the body and at closer distances by moving the head (casting). Together, these results elucidate components of behavioral strategies for odor-based navigation.

Keywords: olfaction, navigation, casting, binaral-olfaction, binaral-sniffing, serial-sniffing

\section{INTRODUCTION}

Mice, insects, and other animals navigate odor trails and locate odor sources with high fidelity, a remarkable feat given the complexity and variability of odor stimuli. Prior studies of olfactory navigation have focused on the mechanisms of navigating through odor plumes in air (Vickers, 2000; Gire et al., 2016) or water (Koehl et al., 2001), or of scent-tracking on the ground, specifically following odor trails (Means et al., 1992; Thesen et al., 1993; Wallace et al., 2002; Porter et al., 2007;

Abbreviations: $(-\mathrm{B}), \mathrm{CSM}$ model without binaral-sniffing component, $k_{\text {binaral }}=0 ;(-\mathrm{C})$, CSM model without concentration-dependent casting, $\sigma=\sigma_{\min } ;(-\mathrm{V})$, CSM model without concentration-dependent velocity, $v=v_{\max } ; \theta$, heading of model body (degrees); $\varphi$, heading of model nose relative to body (degrees); $\sigma_{\max }$, concentration-dependent parameter; $\sigma_{\min }$, concentration-dependent parameter; $\Delta \mathrm{t}$, change in time $(\mathrm{s}) ; \eta_{\mathrm{t}}$, multiplicative uniform noise; $\mid \mathrm{dPhi} / \mathrm{hV}$, curvature; ANOVA, analysis of variance; CRW, correlated random walk; CSM, concentration-sensitive model; $C_{\mathrm{t}}$, concentration magnitude (A.U.); $d_{\text {nares }}$, inter-nostril parameter (cm); IR, infrared; $k$, concentration-dependent parameter; $k_{\text {binaral }}$, binaralolfaction parameter; 1 , nose-to-body length parameter $(\mathrm{cm})$; LED, light emitting diode; OU, Ornstein-Uhlenbeck; $v_{\max }$, maximum velocity parameter $\left(\mathrm{cm} \cdot \mathrm{s}^{-1}\right) ; \mathrm{x}^{\prime}$, derivative $($ of $x) ; x_{\mathrm{n}}$, nose coordinate. 
Khan et al., 2012). However, different strategies may emerge for search under conditions near surfaces where intermittency (the fraction of samples above detection threshold) is high (Connor et al., 2018). In this study, we examine the odor-driven behaviors involved in the search for a discrete odor object under nearsurface, low wind conditions where odor gradient information is likely preserved. Then, we develop a model to investigate multiple potential strategies used for effective odor localization.

One general navigation strategy preserved across species and environmental conditions involves the use of lateral back-andforth changes in orientation of the olfactory sensors during active odor-sampling (Baker et al., 2018). Moths navigating odor plumes use this strategy to reacquire an odor plume, a behavior termed "zig-zagging" or "casting" depending on the magnitude and angle of displacement (Marsh et al., 1978; Kennedy, 1983; Kuenen and Carde, 1994; Carde and Willis, 2008). Flies (Budick and Dickinson, 2006) and cockroaches (Willis et al., 2008) use a similar casting strategy during plume navigation. Lateral backand-forth behaviors are also often observed in animals following odor trails. Rats employ head oscillations that sweep back and forth across a trail as they track it (Khan et al., 2012), a strategy also used by humans (Porter et al., 2007) and ants (Hangartner, 1969; Draft et al., 2018) following trails. In this study, we use the term 'casting' to describe the amplitude of the movement of the olfactory sensors during navigation toward an odor source (Heitz, 2014; Zhao et al., 2017; Baker et al., 2018).

Successful navigation of a complex odor environment likely involves different behavioral strategies under different conditions. Knowledge of the spatial structure of the environment, characteristics of specific odor cues, and general task context may all influence odor-guided behavior. Animals demonstrate strategy-switching between distinct behaviors as a consequence of both changes in odor stimulus properties and task context (Thesen et al., 1993; Pierce-Shimomura et al., 1999; Wesson et al., 2008; Gire et al., 2016). Environmental familiarity and visual cues also influence decision-making strategy during odor source localization. Given visual cues to navigate, exploratory casting based on odor cues disappears on tasks with a small number of potential goal locations; instead, mice begin directly navigating to known odor source locations in straight-line trajectories (Bhattacharyya and Bhalla, 2015; Gire et al., 2016). This resembles the transition from exploration to exploitation behavior observed during successive search trials (Sutton and Barto, 1998; Hills et al., 2015). Thus, both behavioral context and the availability of orienting stimuli are important for strategy choice in odor source localization.

Here, we use an open field odor-based spot-finding task to examine strategies of olfactory search for an odor source deposited on the surface under air flow conditions typical of an indoor setting. By randomizing odor source location, performing experiments in the dark, and using a discrete target odorant object, we prevent mice from switching to methods of systematic search based on memory, visual, or proprioceptive cues (Gire et al., 2016). We find that multiple dimensions of behavior are varied as mice approach the odor source, including velocity and casting, suggesting that these behaviors are associated with odor localization. We then created agent-based models of olfactory search and used simulations to interrogate the roles of binaralsniffing, variable velocity, and variable casting on effective odor localization. Both behavioral and simulation data suggest that multiple behavioral strategies are important for successful odor navigation to a deposited odor source under typical indoor air flow conditions in mice.

\section{MATERIALS AND METHODS}

\section{Ethical Approval}

All experiments were completed in compliance with the guidelines established by the Institutional Animal Care and Use Committee of the University of Pittsburgh.

\section{Animals}

Four male and one female adult M72-IRES-ChR2-YFP knockin mice (The Jackson Laboratory) were used for this study, with training begun after age P47. Average age at training start was P72. All mice were housed singly with behavioral enrichment (running wheel and house) and received unlimited access to water in their home cage.

\section{Behavioral Arena}

A large, open field behavioral arena was used for data collection, as described in Jones and Urban, 2018 (Figure 1A, adapted with permission from Jones and Urban, 2018). The arena is a custom built $36^{\prime \prime} \times 45^{\prime \prime}$ transparent plexiglass and acrylic surface without walls mounted $1.135 \mathrm{~m}$ above the floor in an aluminum frame. Rows of IR light-emitting diodes (LEDs) were mounted along each of the four table surface edges. Data were collected using a camera mounted below the table surface $(1280 \times 1024$ resolution, 11.2 pixels $\cdot \mathrm{cm}^{-1}, 50$ frames $\cdot \mathrm{s}^{-1}$, Flea3, Point Gray Imaging). Trials were run in the dark and LEDs from equipment in the room were blacked out with electrical tape. Remaining light from computer monitors was red-shifted so as to not be visible to the mice.

\section{Odorized Crayon}

We created odorized wax crayons similar to what was described in Jones and Urban (2018). Briefly, $4.9 \mathrm{~g}$ of a Crayola crayon, $0.1 \mathrm{~g}$ of Crayola chalk, and $0.1 \mathrm{~g}$ of odorant consisting of one of three different concentrations $(0.1,1$, and $2 \%$ by weight) of methyl salicylate (Sigma-Aldrich; CAS\#: 119-36-8) diluted in mineral oil (Fisher Chemical Paraffin Oil, 0121-1) were mixed together, and the mixture was poured into a silicon or rubber crayon mold. After $10 \mathrm{~min}$, the mixture cooled and hardened into an odorized crayon.

\section{Task}

Prior to each trial, the table was wiped clean with 200 proof ethanol using a paper towel. After the table dried, a spot $\sim 1 \mathrm{~cm}$ in diameter was drawn in a pseudorandom location in the central area of the table (Figure 1B). Based on recent spot placements, the experimenter attempted to place the spot at a different location on each subsequent trial. For baited trials, a small piece 


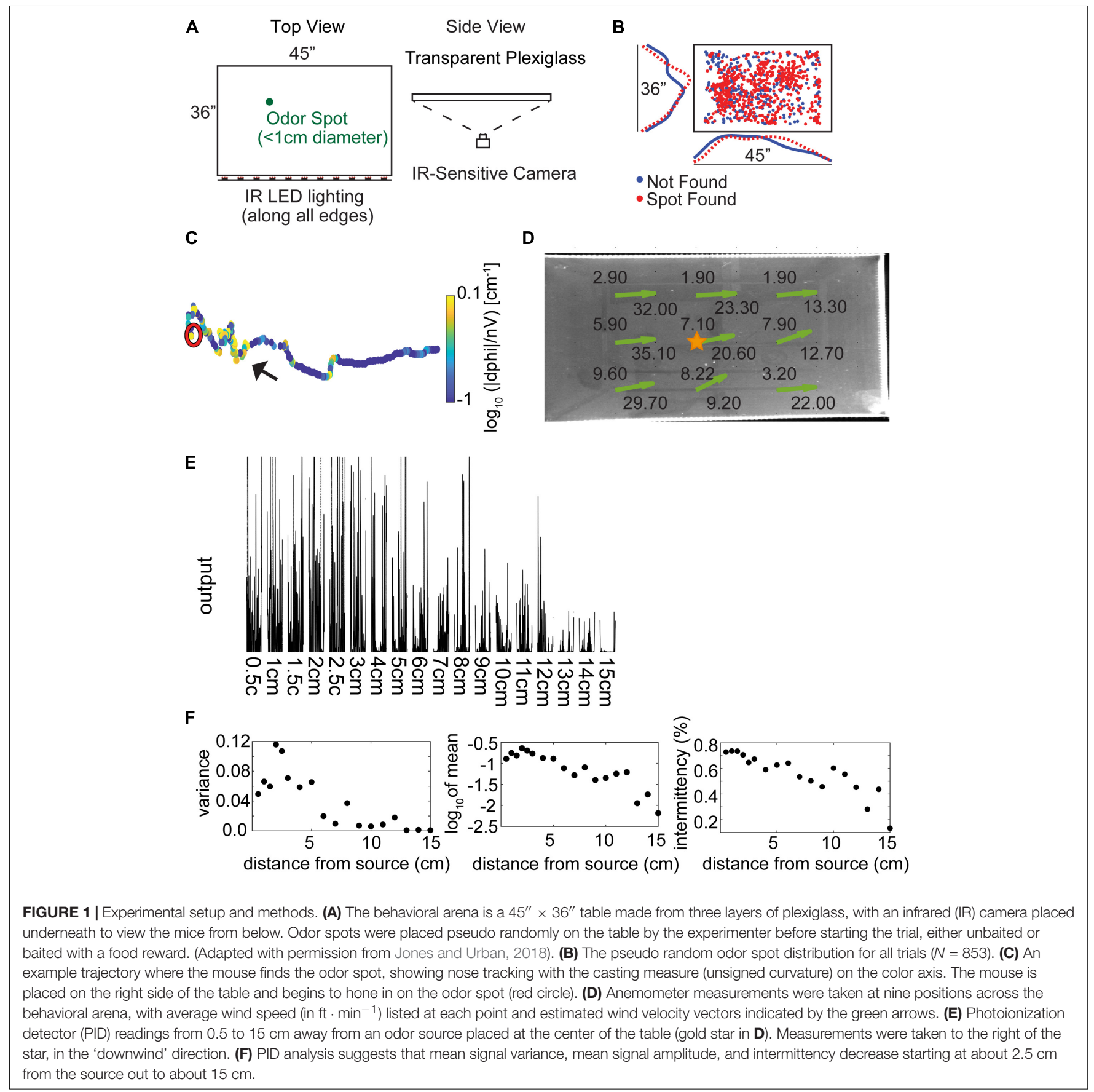

of chocolate or peanut $(\sim 0.1 \mathrm{~g})$ was placed in the middle of the spot, while for unbaited trials, the spot was left empty. No more than 2 unbaited trials were run on a given day to prevent extinction of motivated behavior. With the lights off, a mouse was placed onto the table at a pseudorandom starting location along the right side of the table and allowed to freely explore the arena for approximately $3 \mathrm{~min}$. Over the course of 5 months, 133-197 trials were collected per animal, 3-5 trials per day, 3-5 days per week, at a time between $9 \mathrm{am}$ and $2 \mathrm{pm}$ on a normal light-dark cycle.

\section{Food Deprivation and Task Training}

To motivate mice to perform the task, they were food deprived to $78 \pm 07 \%$ of original body weight. Animals received between 1.8 and $2.2 \mathrm{~g}$ of their normal chow per day and were given free access to water. Animals were trained to associate a spot of odor $(\sim 1 \mathrm{~cm}$ diameter) with food reward, either a small sliver of peanut or milk chocolate. Training consisted of two stages over the course of 7-10 trials: (1) habituation (2-3 days); and, (2) odor-reward association (5-10 days). During habituation, mice were placed on the table for $15 \mathrm{~min}$ and allowed to explore the arena with 
no food reward present. During initial food-odor association, plexiglass dividers were used to decrease the arena size to $25 \%$ of the full arena. A baited odor spot was placed on the table. Once animals found the baited spot in the small arena during at least $66 \%$ of the day's trials, the arena size was increased to $50 \%$ the size of the full arena, followed by an increase to the full $45^{\prime \prime} \times 36^{\prime \prime}$ arena once the spot was again found during $66 \%$ of the day's baited spot trials. Unbaited spot finding trials were introduced only after the animals successfully performed the baited spot task in the full arena. Training ended and data collection began upon successful performance of unbaited spot trials. All reported data are from the full sized arena, but contain both baited and unbaited trials. Baited and unbaited trials analyzed separately were found to be qualitatively similar and so are grouped together.

\section{Video Processing}

Forty-four trials were removed from analysis for technical reasons, leaving 915 trials analyzed. Trials were removed if (1) the video file was corrupted; or, (2) tracking the mouse using Optimouse (see below) was judged by the experimenter to be unsuccessful before data analysis began. This likely occurred due to unintended variation in table illumination on different trials. Of these 915 trials, 54 had odor spot placement within $4 \mathrm{~cm}$ of the edge, and were removed from further analysis due to potential obstruction of mouse trajectories near the spots. Finally, 5 trials were removed because the mouse found the spot within $1 \mathrm{~s}$, indicating that it was initially placed on the arena too close to the odor spot (most trials where this occurred the mouse started within $10 \mathrm{~cm}$ of the odor spot). This left 853 trials analyzed here. Raw avi formatted files were processed using the Optimouse open source program (Ben-Shaul, 2017) to extract body position and nose position. Extracted position files were post-processed with in-house software written in Matlab ${ }^{1}$. Position frames were passed through a 5th order median filter to suppress jitter in the position data and remove high-frequency noise. Position data was excluded if (1) Optimouse failed to detect a position, (2) the $\log _{10}$ of mean mouse brightness was less than 0.5 times the mean for that trial, (3) mean velocity was greater than $100 \mathrm{~cm} \mathrm{~s}^{-1}$, (4) mouse length was greater than $8 \mathrm{~cm}$, (5) mouse position 'jumped' by more than 35 pixels $(3.13 \mathrm{~cm})$ between consecutive frames, and (6) mouse position was $4 \mathrm{~cm}$ or closer from the edge of the arena. Note that the edges of the arena were metal fasteners and the mouse was often partially obscured from below when it walked around the edges. Exclusion criteria (6) accounted for the majority $(N=6 \mathrm{E} 6 ; 84 \%)$ of excluded frames, as mice spent a good deal of time at the edges of the arena. Finally, a nearest neighbor filter was used to remove times when the mouse was stationary, as the computation of orientation of the mouse was unstable here. For the majority of analyses, we focused on either the time before a spot was found, or the full $180 \mathrm{~s}$ for unsuccessful trials. The initial time point in analysis occurred when the mouse's feet were in contact with the surface of the table and the experimenter's hand was withdrawn so as not to be in the field of view of the camera.

\footnotetext{
${ }^{1}$ www.mathworks.com
}

\section{Behavioral Analysis}

A successful spot trial was scored when the mouse's nose came within $1.5 \mathrm{~cm}$ of the spot, based on a sharp increase in the survival function of minimum distance to the spot (Figure 2A).

The fraction of the arena explored was calculated by binning the arena into approximately equal-area parts of 9-10,000 partitions and, then, the percentage of the center of the arena explored (excluding $4 \mathrm{~cm}$ around the edges) was calculated.

Analysis of percent exploration time (or occupancy) entailed defining 100 circular rings around the odor spots at intervals of $1 \mathrm{~cm}$. The number of samples in each ring was then normalized by the total number of samples in the trial to (\% time) and the area of each ring.

The orientation relative to the odor spot was calculated by computing the difference in the four-quadrant arctangent of the vectors from body-to-nose and body-to-spot (Figure 3E).

Derivatives were computed using the adaptive windowing Janabi-Sharifi algorithm (Janabi-Sharifi et al., 2000) available online (Manurung, 2016), but modified to handle missing data points (NaNs) and to allow for post-smoothing. Parameters used were a window size of $50(1 \mathrm{~s})$ and an error term of 0.2 , with post smoothing of $0.1 \mathrm{~s}$.

Casting behavior was quantified by computing the numerical derivative of the arctangent of the nose velocity, taking the absolute value, and then dividing by the linear velocity of the nose $(|\mathrm{dPhi}| / \mathrm{nV})$. This is a modification of the zIdPhi measurement (Papale et al., 2012). $|\mathrm{dPhi}| / \mathrm{nV}$ is equivalent to the signed curvature formula with units of $\mathrm{m}^{-1}$ and is therefore an appropriate measure of tortuosity. The algorithm was extensively tested on variable-frequency sine waves to verify that segments of high curvature were well-separated from segments of high linearity across a wide range of frequencies. Then, for position data from mice, the $\log _{10}$ of the absolute value of $|\mathrm{dPhi}| / \mathrm{nV}$ was taken to generate a roughly normal distribution. An example of the $|\mathrm{dPhi}| / \mathrm{nV}$ casting measure (Figure 1C) for one analyzed behavioral trial shows 'elbows' and angled segments with high curvature (black arrow) well-separated from straight segments of high linearity.

An alternate measure of casting used was the angle of the nose relative to the midline. For this measure, the angle from the nose to the midline was determined by calculating a vector for the mouse's current heading and computing the shortest distance from the detected nose point to that line, then taking the arccosine of that angle.

\section{Anemometer Measurements}

A hot wire anemometer (TSI Velocicalc 9535A) was placed at 9 different positions across the table (Figure 1D). Ten measurements were taken with the sensor aligned to the longaxis or short-axis of the table at each position and the respective averages were taken. We did not generate any airflow during these experiments though air flow occurs in every environment and transport via convection likely dominates the effects of odor diffusion. The room in which these behavioral data were acquired had a ventilation input vent at roughly the center of the room and the primary outflow in the room was a ventilation shaft in the corner. 
A

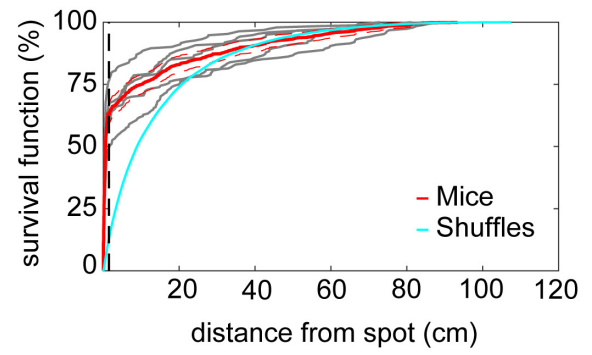

C

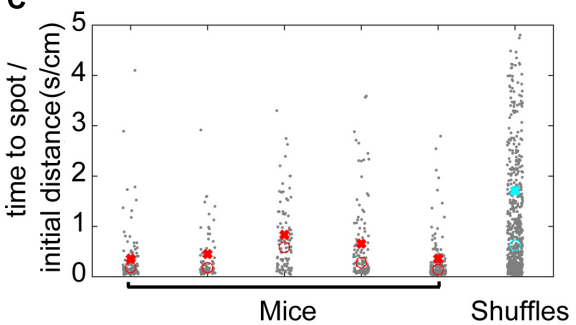

B

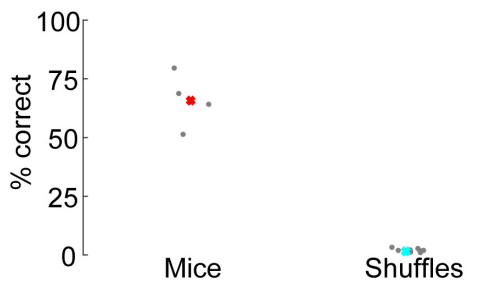

D

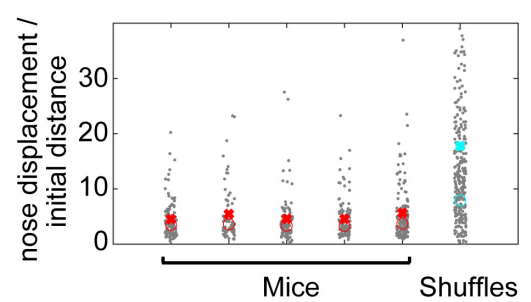

FIGURE 2 | Mice efficiently locate the odor source. (A) The survival function shows the fraction of minimum distances to the spot as a function of distance from the spot. At about $1.5 \mathrm{~cm}$ away from the spot, the average minimum distances start to drop off for mice (red line), suggesting this would be a good threshold distance for counting a trial as successful. In comparison, there is no sharp distinction in the shuffled control data. Instead, shuffled minimum distances tend to increase smoothly as a function of increasing distance. At about $30 \mathrm{~cm}$ away from the odor spot, the survival functions are indistinguishable between mice and shuffled controls. The survival functions of individual mice are plotted to compare inter-individual variability (gray lines). (B) Mice are more successful at finding the odor spots than shuffled controls, suggesting that they are using odor cues to guide their search ( $x=$ mean). (C) The time to spot is divided by the initial distance to the spot, and mice find the spot more quickly than shuffled controls. While statistics were performed on a group level, individual mice are plotted for the purposes of visualizing inter-individual variability $(x=$ mean, $O=$ median; Welch's $t$-test; $p=7.25 \mathrm{E}-18, t$-stat $=8.72$, $\mathrm{df}=1700)$. (D) The nose displacement was divided by the initial distance to the spot, and the relative distance traveled by the nose of mice is less than that of the shuffled controls. While statistics were performed on a group level, individual mice are plotted to give a more precise read out of variability among animals ( $x=$ mean, $O=$ median; Welch's $t$-test; $p=1.2 \mathrm{E}-19, t$-stat $=-9.11$, df $=1573$ ).

\section{PID Measurements}

A cotton swab dipped in 99\% Methyl Salicylate liquid was placed at the center of the table (Figure 1D, gold star) at approximately $0.5 \mathrm{~cm}$ from the surface. Concentration was measured for $30 \mathrm{~s}$ at distances from $0.5 \mathrm{~cm}$ to $15 \mathrm{~cm}$ using a Photoionization Detector (PID) (miniPid, Aurora Scientific). Raw measurements had noise peaks at 60 and $72 \mathrm{~Hz}$, broad spectrum high frequency noise, and substantial baseline drift. To remove the noise peaks, two 2nd order Butterworth IIR notch filters were used. To remove high frequency noise, a (0.2 s backward, $0.02 \mathrm{~s}$ forward) moving mean filter was used. To remove baseline drift, a baseline estimation and denoising function was used (Duval, 2018). The denoised traces (Figure 1E) show large signal fluctuations and, generally, decreasing mean output with distance. Intermittency was calculated as the mean number of points greater than a threshold of 0.005 .

\section{Shuffled Spot Control}

As a control, spots were randomly shuffled and paired with tracking data from one session. Shuffled spots had to be at least $5 \mathrm{~cm}$ away from the original spot. Analyses were repeated with the shuffled control data.

\section{Model Specification}

Based on observations of mouse behavior, we developed an agent-based model that navigated a virtual odor environment.
This agent made temporally discrete sniff-to-sniff comparisons of odor concentration as it moved through virtual space, altering its heading toward higher concentrations and away from lower concentrations. The agent consisted of a body with coordinates $(x, y)$ and moved through space along a heading $\theta$ at a velocity $v_{c}$ :

$$
\begin{aligned}
& x_{\mathrm{t}+\Delta \mathrm{t}}=x_{\mathrm{t}}+v_{\mathrm{c}} \Delta t \cos \theta \\
& y_{\mathrm{t}+\Delta \mathrm{t}}=y_{\mathrm{t}}+v_{\mathrm{c}} \Delta t \sin \theta
\end{aligned}
$$

where $\Delta t=0.1 \mathrm{~s}$ represents a constant the inter-sniff interval. The agent's nose was located at a distance $1=5 \mathrm{~cm}$ from the body at coordinates $\left(x_{n}, y_{n}\right)$. The nose was further divided into left and right nares, $\left(x_{\mathrm{nL}}, y_{\mathrm{nL}}\right)$ and $\left(x_{\mathrm{nR}}, y_{\mathrm{nR}}\right)$, separated by an inter-nares distance, $d_{\text {nares }}=1.8 \mathrm{~mm}$. The two nares are separated by an angle $2 \gamma$. The nose was capable of limited movement independent of the body heading, with an angular deflection $\phi$ relative to the forward heading. Nares positions were given as:

$$
\begin{aligned}
& x_{\mathrm{nL} / \mathrm{nR}}=x+\sqrt{l^{2}+\left(\frac{d_{\text {nares }}}{2}\right)^{2}} \cos (\theta+\phi \pm \gamma) \\
& y_{\mathrm{nL} / \mathrm{nR}}=y+\sqrt{l^{2}+\left(\frac{d_{\text {nares }}}{2}\right)^{2}} \sin (\theta+\phi \pm \gamma)
\end{aligned}
$$

with nose deflection bounded within $\pm \phi_{\max }$ as dictated by mouse anatomy. The agent geometry is shown superimposed on a mouse 

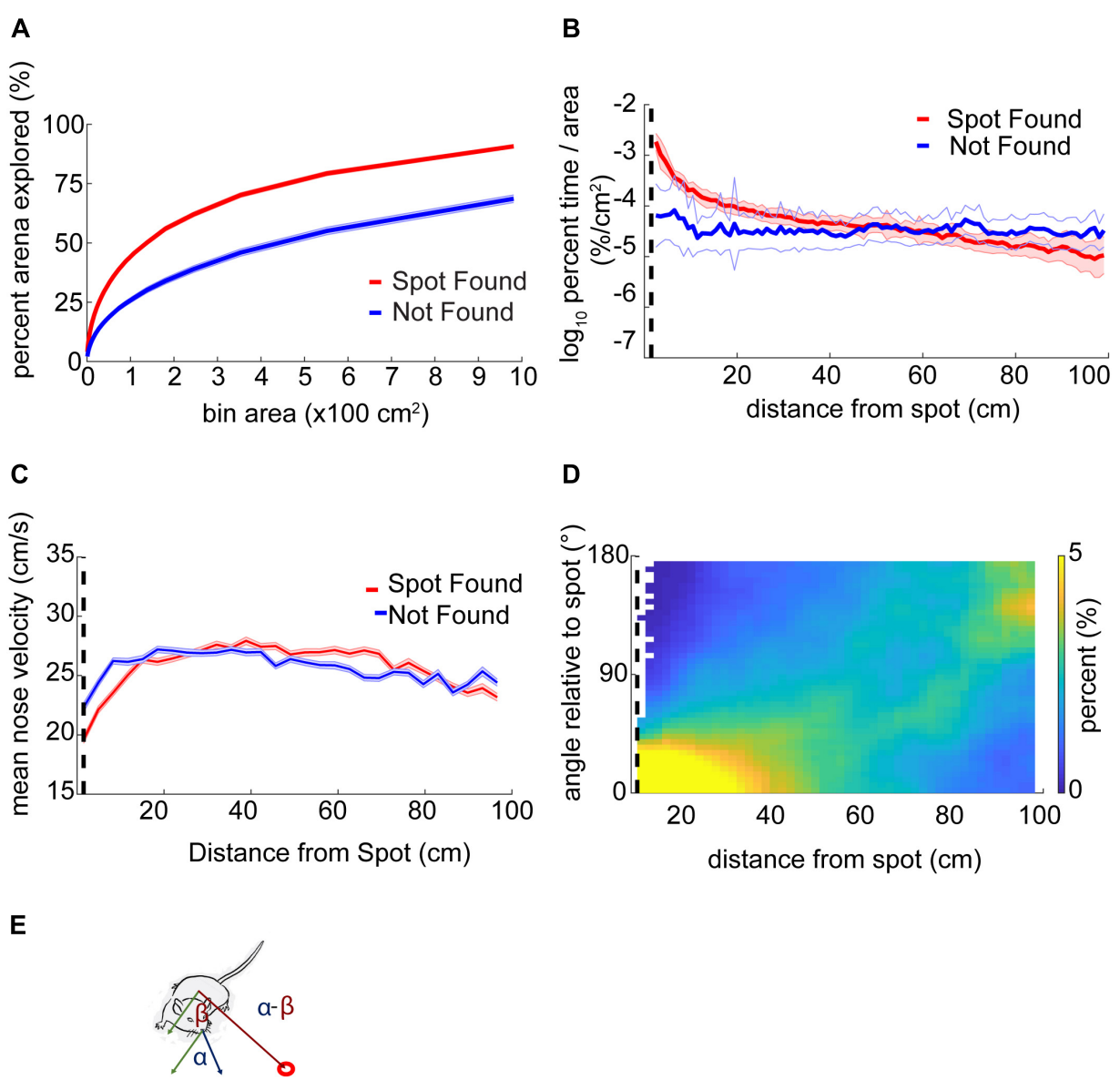

FIGURE 3 | Mouse behavior varies systematically as mice approach the odor source. (A) The center of the arena was broken up into bins of different area and the percentage of those bins visited by mice was counted up and plotted versus the bin area. In general, this 'exploration percentage' increased with increasing bin size while successful trials entailed a higher exploration percentage than unsuccessful trials. (B) The number of samples in 100 concentric rings around the spot was tallied, and normalized by both the total number of samples in a given trial and the area of the ring. This gave a measure of occupancy with units of $\% \cdot \mathrm{cm}^{-2}$. As mice approach the spot on successful trials, their occupancy increases. In contrast, the occupancy on unsuccessful trials remains flat as a function of distance. (C) Mean nose velocity decreases as mice approach the spot on successful trials (ANOVA; $p=2.72 E-91, F=18.73$, df $=28$ ). (D) The orientation of the mouse relative to the odor spot was computed and normalized to $100 \%$ at each distance from the spot. (E) Diagram demonstrating the computation of orientation relative to the spot, with orientation of $0^{\circ}$ indicating the nose is pointing directly at the spot. The angle $\beta$ between the body and spot (red circle) is subtracted from the angle $\alpha$ between the nose and the body.

body (Supplementary Figure S2A) and detailed geometry is shown for clarity (Supplementary Figure S2B).

The independent motion of the nose is what allowed the agent to sample concentrations to the left and right of its current heading and adjust its heading toward higher concentrations (Figure 5C). The distribution of observed mouse nose deflections was approximately Gaussian (Supplementary Figure S2C); therefore, we modeled the angular movement of the nose as an OU process, a mean-reverting CRW with a Gaussian stationary distribution. At each sniff, the nose deflection was updated according to:

$$
\begin{aligned}
\phi_{\mathrm{t}}= & \phi_{\mathrm{t}-\Delta \mathrm{t}}-\frac{\phi_{\mathrm{t}-\Delta \mathrm{t}}}{\tau} \Delta t+d W_{\mathrm{t}} \sqrt{\frac{\Delta t}{\tau}} \\
& +\tanh \left(k_{\text {binaral }}\left(c_{\mathrm{L}}-c_{\mathrm{R}}\right)\right) \Delta t
\end{aligned}
$$

$$
\begin{gathered}
d W_{\mathrm{t}} \sim N\left(0, \sigma_{\mathrm{c}}^{2}\right) \\
\sigma_{\mathrm{c}}=\sigma_{\min }+\left(\sigma_{\max }-\sigma_{\min }\right) \frac{C_{\mathrm{t}}}{k+C_{\mathrm{t}}}
\end{gathered}
$$

where $\tau$ is the characteristic time constant of the process, $\sigma_{c}$ is the concentration-dependent standard deviation of the nose deflection, and $d W_{\mathrm{t}}$ is a normally distributed random variable with mean of zero and standard deviation $\sigma_{\mathrm{c}}$. To this OU process, we add binaral bias in the form of the hyperbolic tangent of the left-right concentration difference; when $C_{\mathrm{L}}>C_{\mathrm{R}}$, the nose is biased to the left and vice versa. Here, $k_{\text {binaral }}$ controls the response of the nose to binaral inputs. The concentration value $C_{\mathrm{t}}$ is the average concentration across both nares at time $t$ :

$$
C_{\mathrm{t}}=\frac{\left(c_{\mathrm{L}}+c_{\mathrm{R}}\right)}{2}
$$


Here, $c_{\mathrm{L}}$ is the concentration at the left naris and $c_{\mathrm{R}}$ is the concentration at the right naris.

We observed that mouse speed increased with distance from the odor source (see Figure 3C). Because the agent in our model does not have information about its distance from the odor source, instead we used a concentration-dependent modulation of speed. Specifically, we use a sigmoidal function with a quartic coefficient to approximate concentration-dependence of speed:

$$
v_{\mathrm{c}}=v_{\max }\left(1-\frac{C_{\mathrm{t}}^{4}}{k+C_{\mathrm{t}}^{4}}\right)
$$

where $v_{\max }$ is the maximum velocity and $k=1 / 2$ concentration units. Note that this is the same $k$ that appears in eq. 7 .

\section{Model Odor Environment}

The agent must iteratively sample concentrations in an odor environment to navigate in the direction of higher concentration. To simulate the odor concentration, $C(x, y)$, we use a noisy bivariate exponential distribution normalized to a value of one at the source:

$$
\begin{gathered}
C(x, y)=e^{-\left(\frac{\sqrt{\left(x-x_{\mathrm{sp}}\right)^{2}+\left(y-y_{\mathrm{sp}}\right)^{2}}}{2 \sigma_{\mathrm{sp}}}\right)}(1+\eta) \\
\eta \sim U\left(-k_{\mathrm{n}}, k_{\mathrm{n}}\right)
\end{gathered}
$$

Here, $\left(x_{\mathrm{sp}}, y_{\mathrm{sp}}\right)$ is the location of the source, $\sigma_{\mathrm{sp}}$ controls the width of the odor distribution around the source (model results were obtained with $\sigma_{\mathrm{sp}}=20 \mathrm{~cm}$ ), and $\eta$ is multiplicative uniform noise added to each point in a spatial grid to approximate the effect of turbulent flow, bounded at $k_{\mathrm{n}}=0.5$.

To represent the table, we generated a static smooth odor landscape grid with a resolution of $1 \mathrm{~mm}$ according to eq. 10 (Figure 5B, step 1). Next, we applied noise according to eq. 11 (Figure 5B, step 2). We then simulated the PID intermittency in the odor signal by randomly setting grid values to zero (Figure 5B, step 3). The probability that a grid value was zeroed, $P_{\text {int }}$, increased with distance from the source according to

$$
P_{\text {int }}=1-e^{k_{\text {int }} \sqrt{\left(x-x_{\mathrm{sp}}\right)^{2}+\left(y-y_{\mathrm{sp}}\right)^{2}}}
$$

where $k_{\text {int }}$ was the decay parameter set to $-0.002 \mathrm{~cm}^{-1}$. Finally, we applied a Gaussian filter with a standard deviation of $4 \mathrm{~mm}$ to introduce local spatial correlations into our landscape (Figure 5B, step 4).

To reflect the apparent lack of mouse orientation toward the source beyond $30 \mathrm{~cm}$, (Figure 3D), the models have a concentration detection threshold of 0.25 concentration units, representing a distance of approximately $30 \mathrm{~cm}$ from the source.

A distribution of simulated spot positions was used, matched to data (Figure 1B), but due to edge effects reducing overall model performance, spots closer than $10 \mathrm{~cm}$ to the walls were not used.

\section{Concentration-Sensitive Model (CSM)}

The agent moves along forward heading $\theta$ at velocity $v_{\mathrm{c}}$, iteratively sniffing the concentration at its nose coordinates
(Eqs. 1-4). At each sniff, the agent's nose randomly samples to the left or right of the forward heading depending on the state of the random nose deflection process (Eqs. 5-7). It compares the current concentration sample, $C_{t}$, to the sample from the previous sniff, $C_{\mathrm{t}-\Delta \mathrm{t}}$. If $C_{\mathrm{t}}$ is greater than $C_{\mathrm{t}-\Delta \mathrm{t}}$, the agent sets its heading to the direction of the nose, $\theta_{\mathrm{t}+\Delta \mathrm{t}}:=\theta_{\mathrm{t}}+\phi_{\mathrm{t}}$. If $C_{\mathrm{t}}$ is less than or equal to $C_{\mathrm{t}-\Delta \mathrm{t}}$, the agent turns away from the direction of the nose, $\theta_{t+\Delta t}:=\theta_{t}-\phi_{t}$. This algorithm is effective at reproducing key results of mouse behavior (Figure 5):

$$
\theta_{\mathrm{t}+\Delta \mathrm{t}}=\theta_{\mathrm{t}}+\phi_{\mathrm{t}} \text { if } C_{\mathrm{t}}>C_{\mathrm{t}-\Delta \mathrm{t}} \text { else } \theta_{\mathrm{t}}-\phi_{\mathrm{t}} \text { if } C_{\mathrm{t}} \leq C_{\mathrm{t}-\Delta \mathrm{t}}
$$

\section{Correlated Random Walk (CRW) Controls}

To test model behavior in the absence of odor-sensitivity, and to serve as a control in case mice were randomly finding the spots, concentration-dependent turning was removed from the model and replaced by a random turning decision. Eq. 13 is replaced by:

$$
\theta_{\mathrm{t}+\Delta \mathrm{t}}=\theta_{\mathrm{t}}+\phi_{\mathrm{t}} \text { if } z>0.5 \text { else } \theta_{\mathrm{t}}-\phi_{\mathrm{t}} ; \quad z \sim U(0,1)
$$

To our surprise, CRW control models had a similar success rate to mice when run for $180 \mathrm{~s}$. After observing mice on videos; however, we determined they were not exploring the arena for the entire $180 \mathrm{~s}$ trial. On successful trials, before finding the odor spots, the average time mice actually spent exploring the center of the arena was only $\sim 30$ s. The CRW controls continuously explored the center of the arena as their decision rule at table boundaries was to bounce off like a billiard ball. Therefore, we ran all simulations for $30 \mathrm{~s}$ to match average mouse exploration time in the center of the arena.

\section{Reduced Model Controls}

To evaluate the effects of different hypothesized features of mouse navigation, we constructed 8 model variant combinations by leaving out binaral-sniffing, concentration-dependent velocity, and/or concentration-dependent casting amplitude. We then evaluated CSM model performance in the absence of these features. To eliminate binaral-sniffing from the model, $k_{\text {binaral }}$ in eq. 5 was set to zero. To remove concentration-dependent velocity from the model, eq. 9 was modified so that $v_{\mathrm{c}}$ was equal to the constant $v_{\text {max }}$. To remove concentration-dependent casting amplitude from the model, eq. 7 was modified so that $\sigma_{\mathrm{c}}$ was set equal to the constant $\sigma_{\min }$. The same 8 model variants were created for the CRW control, and results were averaged together to obtain a control distribution (Figure 6, black lines; Figure 7A, gray bar).

\section{RESULTS}

\section{An Open Field Odor-Based Spot-Finding Task Was Developed}

To examine the behaviors relevant to odor source localization, we designed a task that minimized non-olfactory cues. Five mice were trained to find a $\sim 1 \mathrm{~cm}$ diameter spot of odorized wax within a rectangular behavioral arena of approximately $1 \mathrm{~m}^{2}$ in the dark (Figure 1A, and Methods). Odor sources 
[methyl salicylate with(out) bait] for each of the trials $(N=855)$ across all mice $(N=5)$ were pseudo randomly distributed within the center of the testing table (Figure 1B). An example trajectory shows the nose position of the mouse starting at the right-hand side of the arena and moving leftward before successfully finding the spot (red circle; Figure 1C), with color a measurement of curvature (|dPhi|/nV; see section "Materials and Methods") used to detect casting amplitude. Additional example trajectories are displayed in Supplementary Figure S1, with color indicating relative time (in seconds).

\section{Wind Conditions in the Room}

Airflow measurements were taken on the behavior table (height $1.135 \mathrm{~m}$ above the floor) at 9 positions and 2 orthogonal orientations. Wind speed was measured between $1.9 \mathrm{ft}$. $\min ^{-1}-9.6 \mathrm{ft} \cdot \min ^{-1}$ along the short axis and $9.2 \mathrm{ft} \cdot \min ^{-1}$ $35.1 \mathrm{ft} \cdot \mathrm{min}^{-1}$ along the long axis of the table (Figure 1D). Wind direction was estimated by taking the four-quadrant arctangent (green arrows).

\section{Photoionization Detector (PID) Measurements on Behavior Table}

While attempts to detect a PID signal from the odorized crayon used in experiments were unsuccessful, we still desired an estimate of odor concentration decay as a function of distance from the source. As an approximation to the odorized crayon, we used a cotton swab dipped in $99 \%$ methyl salicylate liquid placed at the center of the table (Figure 1D, gold star) at approximately $0.5 \mathrm{~cm}$ from the surface of the table. The tip of a PID was placed at distances from 0.5 to $15 \mathrm{~cm}$ away from the odor source. After denoising (see section "Materials and Methods") PID traces were highly variable but mean output decreased reliably with distance (Figure 1E). Three aspects of the PID data were characterized. The mean variance increased for distances less than $2.5 \mathrm{~cm}$ and then decreased (Figure 1F, left panel). The increase at small distances could be due to saturation of the PID at high concentrations. The mean output also decreased with distance from the source from about $2.5 \mathrm{~cm}$ (Figure 1F, center panel). Intermittency was measured as the fraction of samples above baseline, and decreased with distance from the source (Figure 1F, right panel).

\section{Mice Learn the Open Field Odor-Based Spot-Finding Task}

To determine an appropriate distance threshold for achieving a successful trial and an approximate minimum concentrationdetection threshold, we examined the survival function with respect to distance from the spot (Figure 2A). For mice, there was a sharp increase in survival at about $\sim 1.5 \mathrm{~cm}$, with a more gradual increase toward $100 \%$ at about $90 \mathrm{~cm}$ from the odor spot (red line). Individual mice varied in their success at $1.5 \mathrm{~cm}$ from about $50-75 \%$ success (gray lines). In contrast, the survival function from shuffled data rose smoothly, crossing the mouse data at about $30 \mathrm{~cm}$ (blue line). We therefore set the distance threshold for finding a spot to be $1.5 \mathrm{~cm}$ and estimated the minimum concentration-detection threshold to be $30 \mathrm{~cm}$ for later modeling purposes.

Given a threshold of $1.5 \mathrm{~cm}$, mice were successful in $64 \%$ $(N=532 / 853)$ of trials (Figure 2B). In comparison, shuffled controls were successful a mere $1.67 \%(N=1,524 / 91,446)$ of trials. Mice had a significantly higher success rate than shuffled controls (Welch's $t$-test; $p=6.22 \mathrm{E}-5, t$-stat $=14.69$, df $=4$ ). A logistic regression on successful versus unsuccessful trials for mouse data revealed several highly predictive explanatory variables (Supplementary Table S1). Baited trials had higher success rates $(p=4.68 \mathrm{E}-12, \beta=2.51)$, potentially indicating higher motivation during these trials or a contribution of the odor of food to localization. Greater arena exploration also predicted success ( $\sim 45 \mathrm{~cm}^{2}$ bins; $p=1.65 \mathrm{E}-15, \beta=15.51$; see Figure 3A). Slower mice $(p=6.89 \mathrm{E}-06, \beta=-0.29)$ were more successful, though they had faster average nose velocity $(p=1.07 \mathrm{E}-04$, $\beta=0.27$ ) and higher casting (integrated $\log _{10}[|\mathrm{dPhi}| / \mathrm{nV}]$ within $30 \mathrm{~cm} ; p=2.56 \mathrm{E}-3, \beta=1.4$ ), suggesting that slower forward body velocity and higher casting amplitude may be features of successful search strategies. Successful completion of this spot-finding task is thus dependent on features of individual trajectories.

We conclude that mice find odor spots above chance levels established by the shuffled control, and predict that further evaluation of specific trajectory features will provide useful information about odor localization strategy.

\section{Mice Efficiently Navigate to the Odor Source in Comparison to Shuffled Controls}

Next, we compared navigation efficiencies of mice to shuffled controls by examining time to spot divided by initial distance and nose displacement divided by initial distance. Time to spot was divided by initial distance to spot, with the average for mice $\left(0.51 \mathrm{~s} \cdot \mathrm{cm}^{-1}\right)$ significantly lower than shuffled controls $(1.71 \mathrm{~s}$. $\mathrm{cm}^{-1}$; Figure 2C; Welch's $t$-test; $p=7.25 \mathrm{E}-18, t$-stat $=8.72$, $\mathrm{df}=1700)$. Then, nose displacement was divided by initial distance to spot, with the average for mice $(4.75 \times)$ significantly lower than shuffled controls $(17.78 \times$; Figure $2 D$; Welch's $t$-test; $p=1.2 \mathrm{E}-19, t$-stat $=-9.11, \mathrm{df}=1573)$. We conclude that mice are more efficient at navigating to the odor source than shuffled controls.

\section{Mouse Behavior Varies Systematically as a Function of Distance From the Odor Source}

As suggested by the logistic regression analysis, features of individual trajectories may be relevant to odor source localization. One potential interpretation of this is that mouse behavior varies as a function of odor concentration along a gradient. Because we did not have direct measurements about the odor gradient being sampled by mice, we used proximity to the odor source as a stand-in.

Using a bin size of $\sim 45 \mathrm{~cm}^{2}$, mice explored an average of $33 \pm 17 \%$ (mean \pm s.d.) of the table before finding the spot. This estimate varied as a function of bin size and successful 
spot-finding (Figure 3A; two-way ANOVA interaction term; $p<1 \mathrm{E}-100, F=83.95, \mathrm{df}=97)$. Mice also explored progressively less of the table with increasing trial number $\left(\sim 45 \mathrm{~cm}^{2}\right.$ bins; ANOVA; $p=2.65 \mathrm{E}-51, F=57.16, \mathrm{df}=5$ ), suggesting refinement of learning throughout the experiment.

We then computed the percentage of time mice occupied at different distances from the odor source, normalized by area (Figure 3B). This revealed a decreasing function with distance on successful trials (red line) compared to a flat distribution with distance on unsuccessful trials (blue line). These lines diverged at a distance of $44 \mathrm{~cm}$ (two sample Kolmogorov-Smirnov test; $p<1 \mathrm{E}-10$ ), suggesting that mice are exploring more within $44 \mathrm{~cm}$ of the odor source on successful trials.

Next, we examined velocity as a function of distance from the odor source (Figure 3C). As mice approached the odor source across all trials, nose velocity slowed down (ANOVA; $p=2.72 \mathrm{E}-91, F=18.73 \mathrm{df}=28$ ), as did body velocity (ANOVA; $p=1.02 \mathrm{E}-160, F=31.12, \mathrm{df}=28)$.

We next examined mouse heading relative to the odor source for successful trials (Figure 3D). If a mouse detects odor cues that provide insight into the location of the odor source, then the mouse's nose should be oriented toward it. At each position sample, the difference in angle between body-to-spot and body-to-nose was computed (Figure 3E). We found that orientation relative to the spot depended on distance from the odor spot (Watson-Williams Test; $p<1 \mathrm{E}-100, F=877.94$, $\mathrm{df}=48$ ), and has a median value not significantly different from zero (i.e., oriented directly at the spot) within $9.54 \mathrm{~cm}$ ( $p>1 \mathrm{E}-3$, circular test for equal medians with Bonferroni correction) (Figure 3D).

In summary, we found that mice vary their search strategy and orient toward the spot as they approach it. Mice also slowed down as they approached the spot. Together, these behavioral changes potentially represent a speed-accuracy trade-off during the final stage of navigation toward an odor object, roughly within $\sim 10-45 \mathrm{~cm}$.

\section{Casting Increases Near the Odor Spot and Is Modulated by Velocity}

We found that curvature increased as mice approached the odor source (ANOVA; $p=2.2 \mathrm{E}-117, F=23.4$, df $=28$ ) with an interaction between distance and successful versus unsuccessful trials (two-way ANOVA; $p=0.0041, F=1.85, \mathrm{df}=28$ ).

We used a stepwise Generalized Linear Model (GLM) to determine the relative effect of velocity and distance on the curvature measure of casting. The first term to include in the stepwise GLM was velocity $(p<1 \mathrm{E}-100$, $F=9636$, Deviance $=1500)$ and the second was distance $(p=7.1 \mathrm{E}-41, F=1481$, Deviance $=1481)$. Non-linear terms were added to the model.

As curvature explicitly depended on inverse nose velocity (see section "Materials and Methods," results from stepwise GLM, and Supplementary Figure S3A), we computed the angle between the head and body centerline as an alternate measure of casting (Supplementary Figure S3B). Using this alternate measure of nose deflection, we again found that casting increased
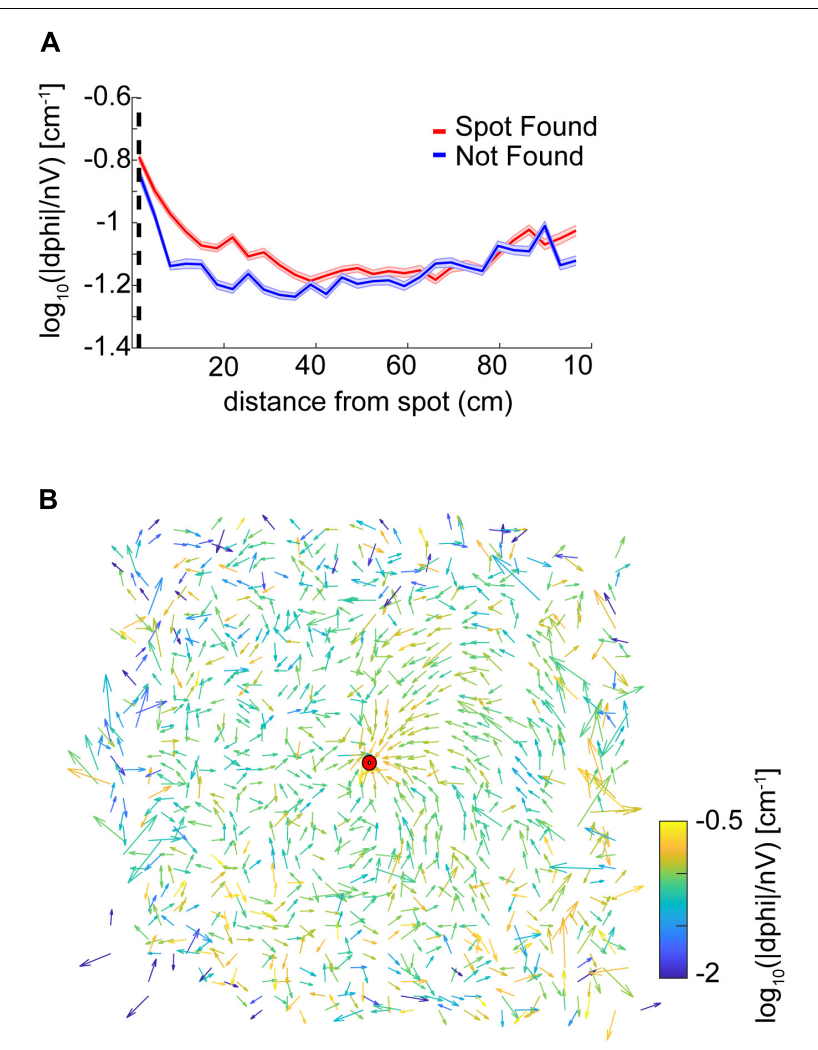

FIGURE 4 | Analysis of casting amplitude using curvature. (A) Unsigned curvature of the nose was computed as a measure of casting amplitude (see section "Materials and Methods") and averaged across successful trials (red line $=$ mean \pm SEM). Casting increased as mice approached the odor spot (ANOVA; $p=1.21 \mathrm{E}-64, F=42, \mathrm{df}=8$ ). (B) The approach vector for successful trials are the vector components of the circular mean of the nose-to-body orientation (angle $\alpha$ in Figure 3E) after centering position data at the odor spots. Vectors are normalized by the circular standard deviation. Color indicates the curvature measure for casting at that point. Most trajectories approach the spot from the northeast.

as mice approach the odor source (ANOVA; $p=2.18 \mathrm{E}-56$, $F=12.82$, $\mathrm{df}=28$, with an interaction between distance and successful versus unsuccessful trials (two-way ANOVA; $p=0.02$, $F=1.61, \mathrm{df}=28)$.

To assess whether animals potentially began using non-odor cues to navigate toward the spots on later trials, we performed one-way ANOVAs on curvature versus binned trial number $(N=6)$. We found an effect of session on curvature (ANOVA; $p=1.42 \mathrm{E}-11, F=12.34, \mathrm{df}=5)$, suggesting that after $60-70$ trials, a shift in strategy occurred which led to systematically less casting. Further analysis revealed that this effect was consistent with systematically increased nose velocity on later trials (ANOVA; $p=9.16 \mathrm{E}-7, F=7.36, \mathrm{df}=5$ ).

We also examined casting amplitude as a function of approach vector. To compute approach vectors, the angle of the nose with respect to the body was taken and positions were centered with respect to the spot, then binned every $2.3 \mathrm{~cm}$. The length of the vector was then normalized by the standard deviation of the angles at that point. The vector colormap indicates 
the $\log _{10}(|\mathrm{dPhi}| / \mathrm{nV})$ at that point. Note that on most trials mice approached the spot from the northeast direction (the 'downwind' direction, see Figure 1D). It was in this direction, at points closest to the source, where casting increased (Figure 4B).

Overall, we found that casting amplitude of mice increases at distances close to the spot, suggesting that it may play a role in the successful navigation to a discrete odor object by mice. This change in casting is potentially correlated with velocity, which also decreases significantly during the approach to the spot.

\section{A Concentration-Sensitive Model (CSM) Was Developed to Capture Aspects of Mouse Behavior Discovered on the Spot-Finding Task}

A typical simulated nose trajectory showing a successful capture displays inter-sniff differences in odor concentration in arbitrary units (Figure 5C). The CSM model used here had parameters: $\Delta t=0.1 \mathrm{~s}, \ell=5 \mathrm{~cm}, v_{\max }=25 \mathrm{~cm} / \mathrm{s}, k=0.5$ concentration units, $\tau=0.1 \mathrm{~s}, \sigma_{\min }=0.2$ radians, $\sigma_{\max }=0.3$ radians, and $k_{\text {binaral }}=200$ The value of $\tau$ chosen gave a good balance of sharp turning nose trajectories with smooth nose trajectories. Values an order of magnitude lower resulted in tight spirals of the nose, while values an order of magnitude higher resulted in loss of maneuverability of the model. $v_{\max }$ was chosen as it was the approximate average velocity of mice. Because simulated odor concentrations were scaled to a maximum of one, $k=0.5$ was chosen as the halfmaximal value for behavioral response. Changing $k$ could be used to 'tune' the model's performance without qualitatively affecting its trajectories. Values of $\sigma_{\max }, \sigma_{\max }$, and $k_{\text {binaral }}$ were chosen so that the model's nose curvature remained qualitatively "mouselike" in character, i.e., the model trajectories did not appear overly tortuous or linear when compared by eye to the mouse trajectories.

To model the observed decrease in velocity close to the odor source, we implemented a concentration-dependent velocity term (Eq. 9). In this term, the maximum velocity, $v_{\max }$ decreases with increasing concentration according to a sigmoidal function. This function was chosen as it was roughly linear in the center of the concentration range and saturated at high concentrations. To model the increase in curvature with approach to the spot, a concentration-dependent widening of the normal distribution was implemented (Eq. 7). These equations were linked by a common parameter $k$ that controlled their rates of change as a function of concentration.

As a control, we created a CRW model that turned independently of concentration (Eq. 14), aligning its heading with its nose $50 \%$ of the time and away from its nose $50 \%$ of the time, resulting in left and right turns in equal proportion. Models using odor cues to navigate should outperform the CRW.

\section{CSM Simulations Have Similar General Properties to Mice}

For simulations, we wished to examine the hypothesis that that mice are (1) moving rapidly where the slope of the gradient is low and detecting changes at different physical locations (a versus b); versus, (2) moving slowly where the slope of the gradient is high and detecting changes by sweeping their heads back and forth (A versus B), which we interpret as casting (Figure 5A).

We implemented a complex odor stimulus model which included (1) generation of a smooth concentration gradient; (2) addition of multiplicative noise which increased near the source; (3) setting pixels equal to zero at a proportion dictated by an intermittency fit such that the farther away from the source the higher the proportion of zeros; and, (4) the smoothing of the resulting stimulus by a two-dimensional local Gaussian filter (Figure 5B). Because we observed a small amount of wind in the room (Figure 1D), plumes would presumably be biased in the direction of the wind along the $y$-axis of the table. Therefore, we tested the effect of removing radial symmetry from the odor stimulus (Supplementary Figure S4A). Removal of radial symmetry along the $y$-axis had negligible effect on simulation performance (Supplementary Figure S4B).

The CSM model with 4-step stimulus generation generally resembled mouse behavior (Figure 5C). Here, concentration difference between consecutive samples is plotted so that the turning away from high-to-low and turning toward low-to-high concentrations is visible. The simulated odor spot is indicated by the red circle.

Spot positions for the CSM and CRW were taken from the mouse data, with spots less than $10 \mathrm{~cm}$ from the edges being removed. The success rate of the CSM was 94\%, significantly greater than the 19\% for CRW controls (Figure 5D; Welch's $t$-test; $p=2.16 \mathrm{E}-22, t$-stat $=59.45, \mathrm{df}=17)$. The CSM therefore successfully used information about concentration to navigate within $1.5 \mathrm{~cm}$ of the odor source.

The nose displacement over initial distance ratio for the CSM $(\sim 5.6 \times)$ was significantly less than the CRW $(\sim 13 \times)$ (Figure 5E; Welch's $t$-test; $p=3.5 \mathrm{E}-11, t$-stat $=-34-41, \mathrm{df}=9)$, indicating that the CSM found the odor spots more effectively than the CRW. The survival function of CSM minimum distance to the spot was shifted to the left and up compared to the CRW control (Figure 5F).

\section{Examining the Structure of CSM Trajectories Suggests That They Approximate Mouse Behavior}

The orientation relative to the spot for the CSM was qualitatively similar to mice (Figure 6A, compare with Figure 3D). The CSM occupancy increased with respect to the CRW occupancy as agents approached the spot (Figure 6B; two-sample Kolmogorov-Smirnov test; $p<1 \mathrm{E}-10$ at $<26 \mathrm{~cm}$ ). This was similar to the occupancy observed in mice on successful trials (Figure 3B). Mean nose velocity of the CSM decreased as agents approached the spot (Figure 6C), as expected, given Eq. 9. On successful trials, the CSM explored less than it did on unsuccessful trials (Figure 6D). This contrasts with the behavior observed in mice (Figure 3A), suggesting that there are unexplored differences between the CSM and mice. CSM casting increased as agents approach the spot (Figure 6E), as expected, given Eq. 7 . This approximates the effect observed in mice (Figure 4A). 
A

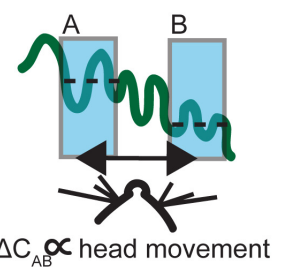

$\triangle \mathrm{C}_{\mathrm{AB}} \propto$ head movement

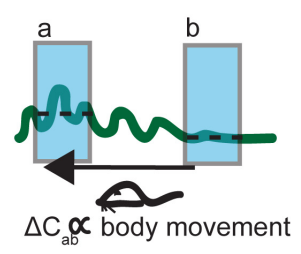

$\Delta \mathrm{C}_{\mathrm{ab}} \propto$ body movement

B

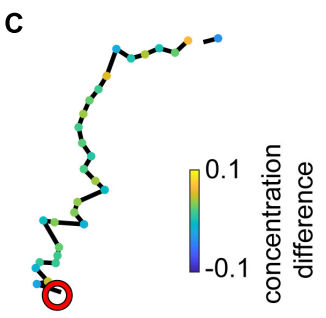

C

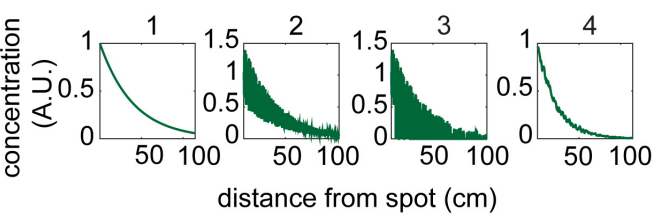

D

E
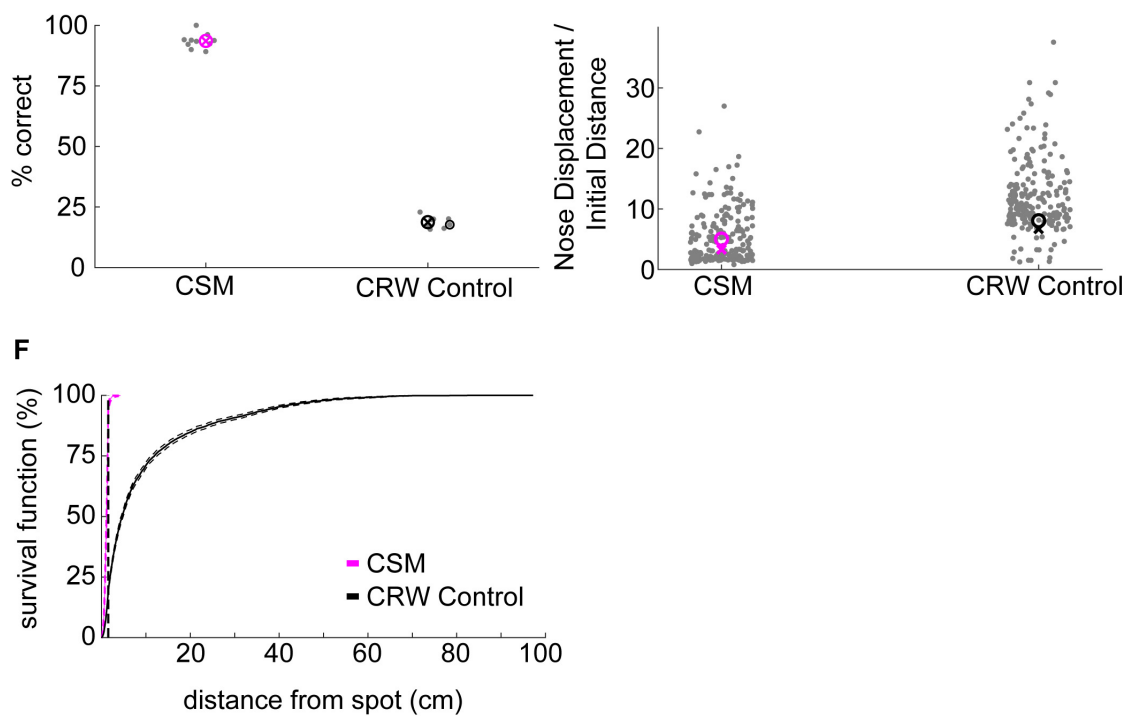

FIGURE 5 | Development of concentration-sensitive model. (A) Our general hypothesis in constructing the model was that lower concentration differences $\left(\Delta C_{a b}\right)$ far from the source would be detectable by gross movement of the body (right panel). In contrast, higher concentration differences ( $\Delta C_{\mathrm{AB}}$ ) would be detectable by fine movement of the head (left panel). In this illustration, the wavy green line represents the concentration of an odorant. The blue shading indicates averaging concentration over an inhalation. The dashed lines indicate the mean concentration for each sniff. (B) The odor concentration profile was constructed in four steps: (1) A smooth concentration gradient was computed; (2) multiplicative random noise was added to the gradient; (3) 'Intermittency' was modeled by setting a proportion of pixels equal to zero; which increased with distance from the source. Finally, (4) Gaussian smoothing was implemented to simulate averaging of the signal over a sniff. (C) An example trajectory from the CSM that successfully found the odor spot (red circle). Concentration difference (A.U.) is indicated. (D) The success rate of the CSM was above 75\%, while the CRW control model had a success rate of below 25\% (Welch's $t$-test; $p=2.16 \mathrm{E}-22, t$-stat $=59.45$, df $=17$ ). (E) The nose displacement divided by the initial distance to the spot was lower for the CSM than for the CRW control $(\mathrm{Welch}$ 's $t$-test; $p=3.5 \mathrm{E}-11, t$-stat $=-34-41$, $\mathrm{df}=9$ ). (F) The survival function of approach distances shows that nearly all CSM trajectories approach within $30 \mathrm{~cm}$ of the source, while CRW control trajectories are more spread out across the entire table, reminiscent of the shuffled control distribution (Figure 2A).

\section{Simulations Allow Separation of Component Strategies for Odor-Driven Navigation}

Simulations allowed for further dissection of the odor-driven factors that impact behavior and successful odor source localization. We created reduced CSMs to examine the relative contributions of three hypothesized behavioral components: binaral-sniffing, concentration-dependent velocity, and concentration-dependent casting amplitude. While all reduced model variants outperformed CRW controls (Figure 7A), removal of any of these behavioral components decreased model success. The removal of concentration-dependent velocity led to the largest change in success rate $(-\mathrm{V}, \Delta 30 \%)$, followed by concentration-dependent casting amplitude (-C, $\Delta 8 \%)$, and binaral-sniffing $(-\mathrm{B}, \Delta 3 \%)$. The change in success rate was significant for removal of concentration-dependent velocity (Welch's $t$-test; $p<1 \mathrm{E}-100, t$-stat $=169$, df $=1472$ ), concentration-dependent casting (Welch's $t$-test; $p<1 \mathrm{E}-100$, $t$-stat $=58.3$, df $=1759$ ), and binaral sniffing (Welch's $t$-test, $p=2.65 \mathrm{E}-100, t$-stat $=22.6, \mathrm{df}=1927)$. The effect size depended on the number of bootstrapped samples (out of 100,000) and groups in each sample. For this analysis, we chose 1000 groups with 10,000 samples in each group, selected without replacement. 


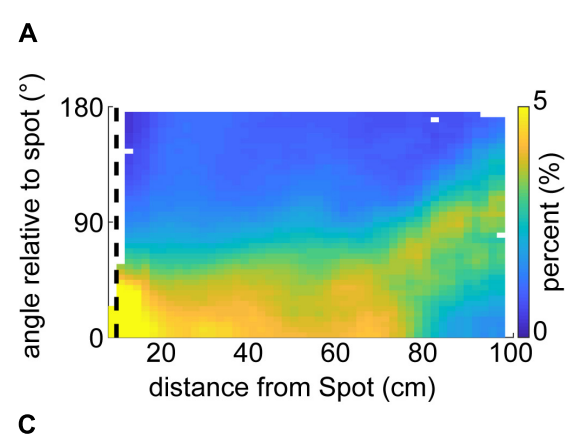

\section{B}
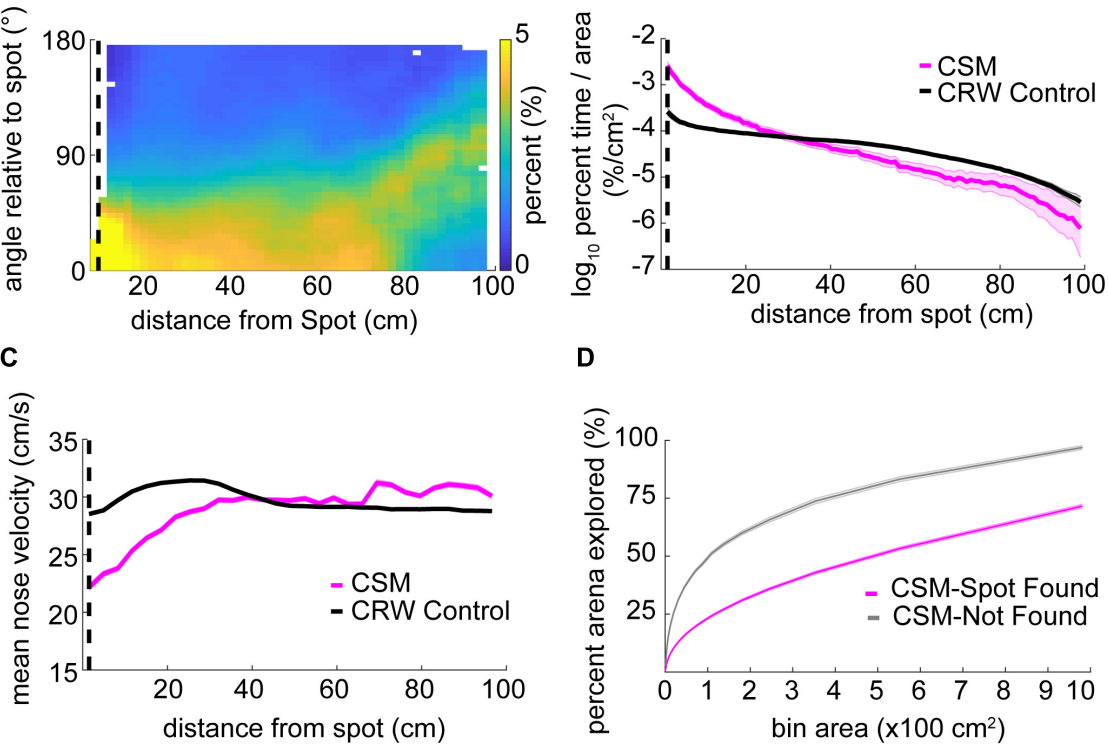

D
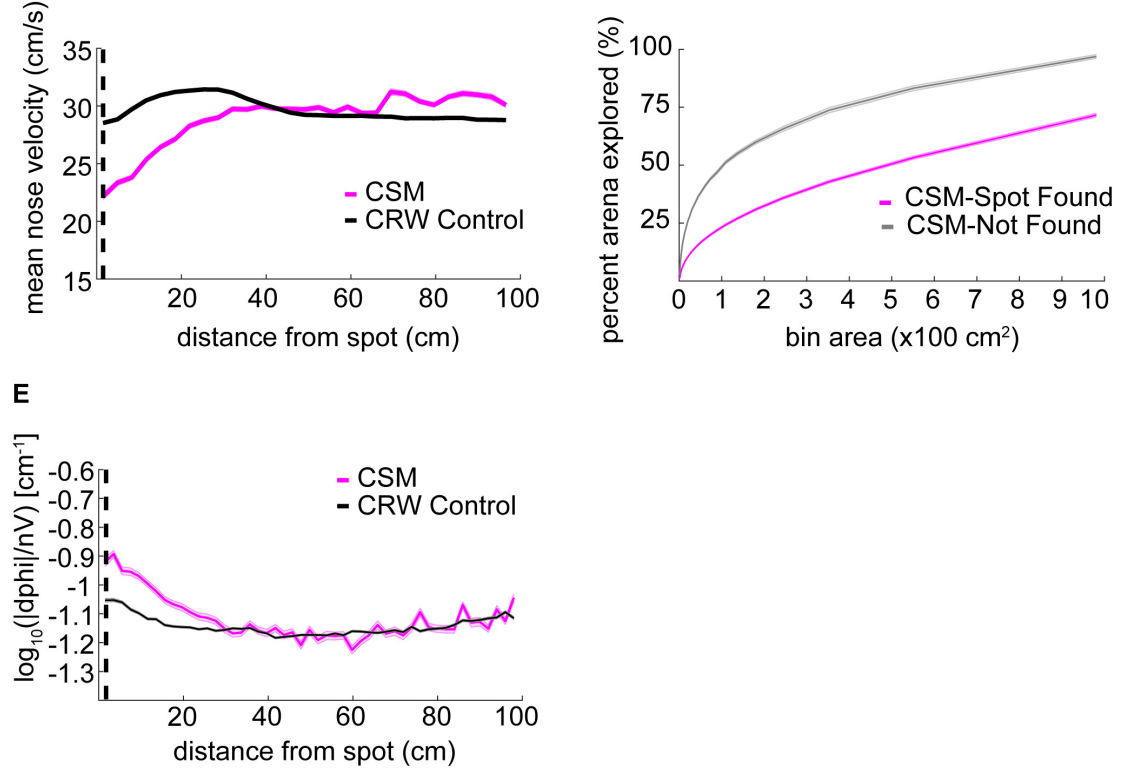

Detailed analysis of CSM trajectories. (A) The orientation of the CSM model relative to the (B) The occupancy of the CSM resembled that of mice (magenta line; compare red line Figure 3B), and significantly diverged from the CRW control (gray line) at $\sim 26 \mathrm{~cm}$ from the odor spot (two-sample Kolmogorov-Smirnov test; $p<1 \mathrm{E}-10$ at $<26 \mathrm{~cm}$ ). (C) The mean nose velocity of the CSM (magenta line) decreased as the model approached the odor spot, consistent with what was observed with mice (compare red line Figure $\mathbf{3 C}$ ). The nose velocity of the CSM was different from the CRW control (black line). (D) In contrast to mice, successful trials of the CSM (magenta line) showed less exploration than unsuccessful trials (gray line) (compare red line to blue line in Figure $\mathbf{3 A}$ ). (E) Casting increased as the CSM approached the spot (magenta line).

These behavioral components of olfactory search are synergistic; simultaneous removal of two or more of them leads to a greater reduction in success rate than removal of individual components. Model variant $(-\mathrm{VC})$, in which both velocity decrease and casting increase are removed, resulted in a significant $\Delta 12 \%$ change in model success relative to $(-\mathrm{V})$ alone (Welch's $t$-test; $p<1 \mathrm{E}-100, t$-stat $=-55.9, \mathrm{df}=1997$ ). All groups performed significantly better than the CRW Control, even with all three components of the model removed (-VBC; Welch's $t$-test; $p<1 \mathrm{E}-100, t$-stat $=67.08, \mathrm{df}=1997)$. This is logical since ( $-\mathrm{VBC})$ still makes decisions based on inter-sniff concentration differences, while the CRW Control makes random decisions.

Significance of model parameters was also established by shuffling 12 parameters of the model $50 \mathrm{k}$ times, and comparing the effect of each parameter using a logistic regression (Supplementary Table S2). We also included two dependent variables from the model trajectories that we thought might impact success rate, the mean casting $\left(\log _{10}(|\mathrm{dPhi}| / \mathrm{nV})\right.$ within
$30 \mathrm{~cm}$, and the mean nose velocity within $30 \mathrm{~cm}$. Note that for this analysis, we split $k$ into separate parameters for casting $\left(k=k_{\mathrm{c}}\right.$ in Eq. 7) and velocity ( $k=k_{\mathrm{v}}$ in Eq. 9). Likewise, we labeled and varied the power parameters $\left(n_{\mathrm{c}}=1\right.$ in Eq. 7$)$ and $\left(n_{\mathrm{v}}=4\right.$ in Eq. 9) from 0 to 5 . We also added a coefficient in front of the tanh binaral term in Eq. 5 ( $\left.k_{\text {binaral } 2}\right)$, and $k_{\text {binaral }}$ in Eq. 5 is

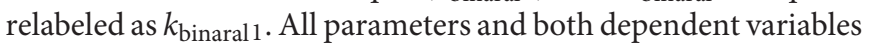
significantly impacted success rate, except for the parameters $\tau$, $\sigma_{\min }$, and $k_{\mathrm{c}}$.

To more rigorously test the effects of different levels of noise, the noise level $k_{\mathrm{n}}$ in Eq. 11 (also see step 2 in Figure 5B) was varied from 0 to 1 and the Gaussian smoothing $(4 \mathrm{~mm}$ in reported CSM variant; step 4 in Figure 5B) was varied from 0 to $10 \mathrm{~mm}$. Success rate rapidly dropped off as $k_{\mathrm{n}}$ approached 1 or smoothing approached $0 \mathrm{~mm}$ (Figure $7 \mathbf{B}$ ).

To more rigorously test the effects of the 2-parameter binaral term, we re-ran the 6 reduced model variants 100,000 times each with a coefficient of $k_{\text {binaral } 2}=3$ in front of the tanh term in Eq. 5 . 


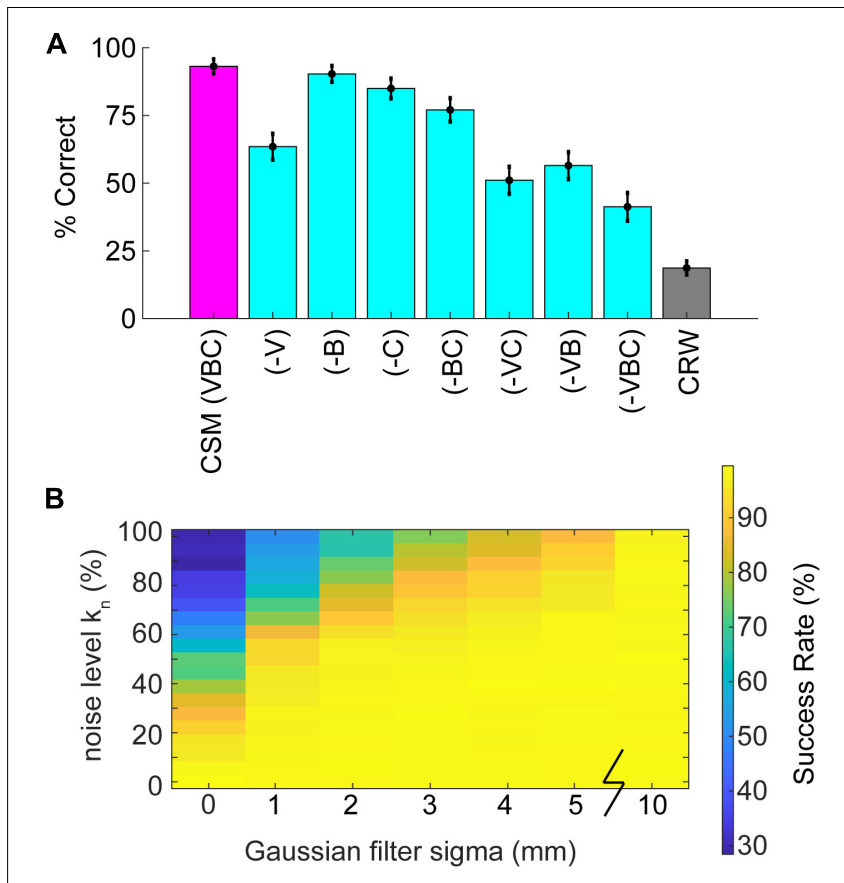

C

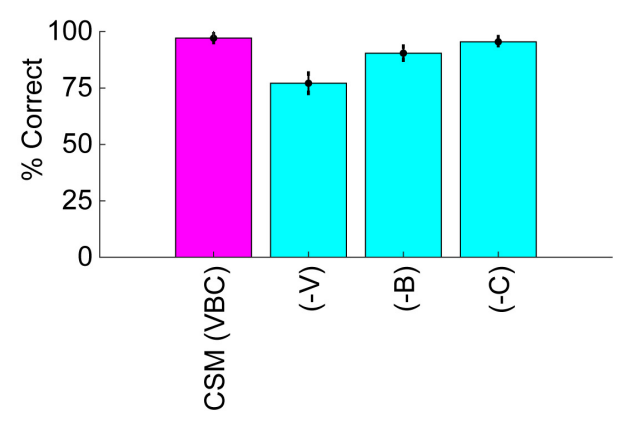

FIGURE 7 | Analysis of reduced models and parameter sweeps. (A) The full model (CSM) included three components: (1) a concentration-dependent velocity term ( $\mathrm{V}$, (2) a concentration-dependent casting term (C); and, (3) a binaral-olfaction term (B). Removal of any of these components $(-V),(-C)$, or (-B) may, in principle, reduce model performance. (B) Comparison of noise effects on CSM success rate shows that the CSM model is robust for a wide array of different noise conditions, but fails if random noise approaches 100\% or Gaussian filtering approaches $0 \mathrm{~mm}$. (C) We added a coefficient in front of the hyperbolic tangent term in Eq. 5 and set it equal to 3 . With this additional term, (-B) had a larger effect on performance; however, the effect of removing $(-\mathrm{V})$ or $(-\mathrm{C})$ terms decreased.

Again, we chose 1,000 groups with 10,000 samples in each group, selected without replacement. This resulted in smaller effects of removing $(-\mathrm{V})$ and $(-\mathrm{C})$, but a larger effect of removing $(-\mathrm{B})$, suggesting that strong binaral-sniffing may be able to compensate for removal of variable velocity and casting (Figure 7C).

In summary, reduced models revealed that individual components of the overall strategy worked together to maximize task performance, with concentration-dependent velocity and casting having the greatest impact on successful task performance.

\section{DISCUSSION}

Here, we described the navigation strategies of mice during an open field odor-based spot-finding task. Over the course of 7-13 days (3-5 sessions/day), mice could be trained to perform this task on a $45^{\prime \prime} \times 36^{\prime \prime}$ open field arena. Performance of mice exceeded that of shuffled controls, indicating that mice were using odor cues to localize the odor target. Olfactoryguided behavior changed as a function of distance; specifically, (1) nose velocity decreased close to the source, (2) casting increased close to the source, and, (3) occupancy increased close to the source. Mice demonstrated reliable spot-finding within $10-45 \mathrm{~cm}$ of the odor source. The Concentration Sensitive Model (CSM) was built to capture these observations by incorporating concentration-dependent velocity and casting terms, as well as a postulated binaral comparison term. Removing components of the CSM demonstrated that each had a significant impact on performance.

\section{There Is a Potential Speed-Accuracy Trade-Off in Search Strategy Close to the Odor Spot}

As mice approach an odor source, they slow down and increase their exploration time in a smaller annulus around the spot. This is similar to observations in fruit flies following a plume upwind to an odor source (Budick and Dickinson, 2006). We suggest this phenomenon may be a type of speed-accuracy tradeoff (Heitz, 2014) in olfactory search behavior. Specifically, when concentration reaches some perceptual threshold, mice may "switch" into a local search strategy designed to increase accuracy at the expense of speed to target. In this local search strategy, the mouse spends more time in regions of high concentration where it is more likely to hit its target. In agreement with this hypothesis, elimination of concentration-dependent velocity decrease led to less success in reduced models (Figure 7A). While it is tempting to ascribe all behavioral output on this task to the salience of the odor cues, mice may fall back on coordination of olfactory cues with other input modalities, especially as they get closer to the odor source. For example, they may integrate olfactory cues with secondary cues from somatosensory or proprioceptive inputs.

\section{Binaral-Sniffing May Facilitate Search Near the Odor Source}

Use of binaral-cues for navigation has been observed in moles (Catania, 2013), snakes (Schwenk, 1994), and insects (Martin, 1965; Steck et al., 2010) suggesting it may be an evolutionarily conserved strategy. In mammals, neurons in the anterior olfactory nucleus may play a key role in integration of bilateral stimuli that are key to binaral-sniffing (Kikuta et al., 2010) as this is one of the earliest locations in which information from both left and right naris can be integrated. Once a trail's direction or the rough direction to a spot is determined, binaral-sniffing cues may be useful for determining the direction to cast in order to continue following the direction of the trail (Khan et al., 2012), or steer toward the odor spot. Binaral-sniffing allowed the CSM to 
preferentially cast in the direction of the odor source only when the odor gradient was large (i.e., near the source). Removal of binaral-sniffing led to a statistically significant decrease in CSM success (Figure 7C), an observation compatible with the model developed by Catania (2013), where binaral-sniffing becomes a relevant strategy on steep concentration gradients.

Future research may wish to examine if effective nares separation $\left(d_{\text {nares }}\right)$ determined by parameters such as inhalation volume, flux, and gradient rather than the distance between the nares. For example, inhalation in rats is largely from the lateral direction, rather than the front of the animal (Wilson and Sullivan, 1999). Pressure differences generated during sniffing inhalation at different magnitudes and frequencies (slow deep breath versus rapid sniffing) may also draw in relatively different concentrations of molecules from different distances away from the nares.

\section{Odor Source Localization May Rely on Cognitive Mapping}

Navigation to an odor source with serial-sniffing is probably intimately tied to navigation through space using the cognitive map (Jacobs, 2012). Specifically, the distance traveled between the first to the second sniff in the concentration comparison helps determine an updated heading toward the source or along the trail. In support of this view, hippocampal place cells may change their firing patterns (called remapping) to match the relocation of an orienting odor source in a circular arena (Zhang and Manahan-Vaughan, 2015). Thus, serial-sniffing is potentially more relevant to gross odor localization than binaral-sniffing. For example, to determine trail direction using binaral-sniffing most efficiently, an animal would have to be oriented roughly perpendicular to the trail so that its nares are sensing maximally different concentrations. In contrast, studies with dogs repeatedly find that serial-sniffing of 2-5 footprints is required to determine a trail's direction (Thesen et al., 1993; Hepper and Wells, 2005). Similarly, initial odor source seeking may utilize serial-sniffing to quickly map larger spatial areas, including eliminating subspaces where no odor is detected (Vergassola et al., 2007).

\section{Casting Is Related to Olfactory-Guided Navigation to a Discrete, Static Odor Source}

Casting has been described as "zig-zagging" behavior in moths flying upwind during pheromone-tracking (Charlton et al., 1993), and has been reported to occur outside of an odor plume when a moth is attempting to re-acquire the odor (Kennedy, 1983). Head-scanning behaviors, also called casting, have been observed in Drosophila larvae, rats, moles, and humans (Baker et al., 2018). Here we find that mouse casting (or head scanning) amplitude increases during approach to the odor source. The moth data suggest that casting for edge detection increases whenever there are intermittent gaps in the odor signal, as they cast to re-acquire the odor plume. If this were true for mice performing the open field odor-based spot-finding task, we would expect casting to be higher farther away from the odor source where the signal is sparse. In fact, we find the opposite. Casting in moths and head scanning/casting in other animals may therefore be mediated by different types of mechanisms and occur for different purposes.

\section{Complexities to Address in Future Studies Include Decision-Rules and Individual Variability in Strategy Utilization}

Our data suggest that mice may use multiple search strategies during an open field odor-based spot-finding task. We speculate that the relative contribution of strategies may also be variable between individuals, as has been observed during fruit fly navigation during odor presentation (Tao et al., 2019). This may mean a specific instantiation of our model (with one set of parameters) captures behavior for one individual mouse, but fails to capture behavior for some other subset of mice. For example, the $k$ parameter could be used to 'tune' aspects of the model's trajectories which may better fit the data for a subpopulation of mice. A much larger cohort of animals than was used here would be necessary to evaluate this idea; however, the general principle is that similar-looking ethologically relevant behavior can emerge from different model permutations.

A limitation of the current model is that it incorporates an 'all or nothing' decision at each sniff. It may be more appropriate to use a probabilistic decision rule to combine binaral- and serial-sniffing into a unified output. Also, a model where pausing emerges during epochs of uncertainty in sampling of the odor concentration may be advantageous. Additionally, the model is currently limited to detection of one odorant-it would be useful to distinguish different features of diverse odorants. Finally, stimulus models with 'zeros' distributed throughout the simulated odor landscape produced high failure rates. Developing a model that is robust to this type of intermittency would be a logical next step.

While the CSM is capable of finding the odor source when the odor is consistently above threshold, it does not attempt to capture mouse behavior where the odor concentration is below detection threshold. The mouse may, however, be engaging in more complex navigation behavior here, including mapping of the odor environment. For example, the model of Vergassola et al., 2007 builds a cognitive map of odor source location likelihood; it utilizes both sparse odor detection events and frequent subthreshold observations to update its map of source location likelihood.

In summary, we discover that mice vary their search strategy during navigation toward a discrete odor object, modulating features of behavior as a function of distance from the target. A CSM successfully reproduces mouse behavior, while allowing for the analysis of the relative contributions of different putative components of navigation. Future work is needed to experimentally dissect how these variables causally impact odordriven navigation.

\section{DATA AVAILABILITY STATEMENT}

The datasets generated for this study are available on request to the corresponding author. 


\section{ETHICS STATEMENT}

The animal study was reviewed and approved by the Institutional Animal Care and Use Committee (IACUC) of University of Pittsburgh.

\section{AUTHOR CONTRIBUTIONS}

$\mathrm{JH}, \mathrm{AL}, \mathrm{BE}$, and $\mathrm{NU}$ designed the behavioral experiments. AP, JH, $\mathrm{BE}$, and NU designed the simulations. $\mathrm{AL}$ and $\mathrm{KP}$ conducted the behavioral experiments. AP, JH, and AL analyzed the data. AP and $\mathrm{JH}$ wrote the MATLAB code. AP calculated the statistics. AP, $\mathrm{JH}, \mathrm{AL}, \mathrm{BE}$, and NU wrote the manuscript.

\section{FUNDING}

This work was supported by the National Science Foundation (1555862 to NU and 1555916 to BE); the National Institutes of Health, National Institute on Deafness and Other Communication Disorders (F30DC015161 to AL), and the Pennsylvania Department of Health's Commonwealth Universal Research Enhancement Program (NU).

\section{ACKNOWLEDGMENTS}

We thank Gregory LaRocca for technical help, and members of the Urban laboratory as well as Yu Chen, Robert Kass, Nour Riman, Erin Connor, and John Crimaldi for helpful discussions. We thank Kathy Nagel and Jonathan Victor for helpful comments on an earlier draft of the manuscript. A special thanks to Peter Jones for designing and building the behavior table used in this experiment. Thanks to Chris D. Rand from Aurora Scientific for technical help with the PID.

\section{REFERENCES}

Baker, K. L., Dickinson, M., Findley, T. M., Gire, D. H., Louis, M., Suver, M. P., et al. (2018). Algorithms for olfactory search across species. J. Neurosci. 38, 9383-9389. doi: 10.1523/JNEUROSCI.1668-18.2018

Ben-Shaul, Y. (2017). OptiMouse: a comprehensive open source program for reliable detection and analysis of mouse body and nose positions. BMC Biol. 15:41. doi: 10.1186/s12915-017-0377-3

Bhattacharyya, U., and Bhalla, U. S. (2015). Robust and rapid air-borne odor tracking without casting. eNeuro 2:ENEURO.102-ENEURO.115. doi: 10.1523/ ENEURO.0102-15.2015

Budick, S. A., and Dickinson, M. H. (2006). Free-flight responses of drosophila melanogaster to attractive odors. J. Exp. Biol. 209(Pt 15), 3001-3017. doi: 10. 1242/jeb.02305

Carde, R. T., and Willis, M. A. (2008). Navigational strategies used by insects to find distant, wind-borne sources of odor. J. Chem. Ecol. 34, 854-866. doi: 10.1007/s10886-008-9484-5

Catania, K. C. (2013). Stereo and serial sniffing guide navigation to an odour source in a mammal. Nat. Commun. 4:1441. doi: 10.1038/ncomms 2444

Charlton, R. E., Kanno, H., Collins, R. D., and Carde, R. T. (1993). Influence of pheromone concentration and ambient-temperature on flight of the
This manuscript has been released as a Pre-Print at bioRxiv (Liu et al., 2020).

\section{SUPPLEMENTARY MATERIAL}

The Supplementary Material for this article can be found online at: https://www.frontiersin.org/articles/10.3389/fnins. 2020.00218/full\#supplementary-material

FIGURE S1 | Example traces. Successful trials (left and center panels) have the time to spot indicated by the text. The relative time of each position sample is indicated by the color. Unsuccessful trials (right panel) have the total trial time indicated by the text. Color represents the relative time of each position sample. Spot positions are indicated by red circles.

FIGURE S2 | Detailed model geometry. (A) Model specification of the body position $(x, y)$, and the body angle $\theta$ superimposed on a diagram of the mouse. The nose position $\left(x_{n}, y_{n}\right)$ is the mean position between the left and right nares. (B) Nose angle $\phi$ (gray dashed line) is the angle between $\theta$ and nose position, and is constrained by a maximum angle $\phi_{\max }$. The head-body length I is the distance between the body position and the nose position, which is sub-divided into two nostrils separated by a distance $d_{\text {nares }}$. The angle from the center of the nose to the edge of the nostril is $\gamma$. (C) The distribution of mouse nose deflection angles $\phi$ (blue bars) is approximately Gaussian \{dashed red line; $a^{*} \exp \left[-((x-b) / c)^{\wedge} 2\right]$; $a=0.04, b=0.06, c=1.92\}$.

FIGURE S3 | Additional casting analyses. (A) Curvature as measured by $\log _{10}$ $(\mid \mathrm{dPhi} / \mathrm{nV})$ depends on nose velocity. The color map indicates the percentage of samples in each bin. (B) Averaged across successful trials, nose deflection angle $\phi$ increases as the distance to the spot decreases (line = mean \pm SEM; ANOVA; $p=2.18 \mathrm{E}-56, F=12.82, \mathrm{df}=28)$.

FIGURE S4 | Non-symmetric plume analysis. (A) To test the effect of removing radial symmetry on model performance, we created elliptical sources using an additional shape parameter $\alpha$. Increasing the shape parameter resulted in more longitudinally skewed plumes. (B) Performance was not strongly affected by removing the assumption of radial symmetry.

TABLE S1 | Logistic regression analysis on spot-finding success in mice. Significant variables in bold.

TABLE S2 | Logistic regression analysis of CSM parameters on spot-finding success. Significant parameters are in bold.

gypsy-moth, lymantria-dispar (L), in a sustained-flight wind-tunnel. Physiol. Entomol. 18, 349-362. doi: 10.1111/j.1365-3032.1993.tb00608.x

Connor, E. G., McHugh, M. K., and Crimaldi, J. P. (2018). Quantification of airborne odor plumes using planar laser-induced fluorescence. Exp. Fluids 59:137. doi: 10.1007/s00348-018-2591-3

Draft, R. W., McGill, M. R., Kapoor, V., and Murthy, V. N. (2018). Carpenter ants use diverse antennae sampling strategies to track odor trails. J. Exp. Biol. 221:jeb185124. doi: 10.1242/jeb.185124

Duval, L. (2018). BEADS: Baseline Estimation And Denoising with Sparsity, version 1.7.0.1. Available at: https://www.mathworks.com/matlabcentral/fileexchange/ 49974-beads-baseline-estimation-and-denoising-with-sparsity (accessed March 3, 2020).

Gire, D. H., Kapoor, V., Arrighi-Allisan, A., Seminara, A., and Murthy, V. N. (2016). Mice develop efficient strategies for foraging and navigation using complex natural stimuli. Curr. Biol. 26, 1261-1273. doi: 10.1016/j.cub.2016.03.040

Hangartner, W. (1969). Structure and variability of the individual odor trail in Solenopsis geminata Fabr (Hymenoptera formicidae). Z. Vgl. Physiol. 62, 111-120. doi: 10.1007/bf00298046

Heitz, R. P. (2014). The speed-accuracy tradeoff: methodology, and behavior. Front. Neurosci. 8:150. doi: 10.3389/fnins.2014.00150 
Hepper, P. G., and Wells, D. L. (2005). How many footsteps do dogs need to determine the direction of an odour trail? Chem. Sens. 30, 291-298. doi: 10. 1093/chemse/bji023

Hills, T. T., Todd, P. M., Lazer, D., Redish, A. D., Couzin, I. D., and Grp, C. S. R. (2015). Exploration versus exploitation in space, mind, and society. Trends Cogn. Sci. 19, 46-54. doi: 10.1016/j.tics.2014.10.004

Jacobs, L. F. (2012). From chemotaxis to the cognitive map: the function of olfaction. Proc. Natl. Acad. Sci. U.S.A. 109, 10693-10700. doi: 10.1073/pnas. 1201880109

Janabi-Sharifi, F., Hayward, V., and Chen, C. S. J. (2000). Discrete-time adaptive windowing for velocity estimation. IEEE Trans. Control Syst. Technol. 8, 10031009. doi: $10.1109 / 87.880606$

Jones, P. W., and Urban, N. N. (2018). Mice follow odor trails using stereo olfactory cues, and rapid (sniff)to sniff comparisons. bioRxiv [Preprint]. doi: 10.1101/ 293746

Kennedy, J. S. (1983). Zigzagging and casting as a programmed response to windborne odor - a review. Physiol. Entomol. 8, 109-120. doi: 10.1111/j.1365-3032. 1983.tb00340.x

Khan, A. G., Sarangi, M., and Bhalla, U. S. (2012). Rats track odour trails accurately using a multi-layered strategy with near-optimal sampling. Nat. Commun. 3:1712. doi: $10.1038 /$ ncomms 1712

Kikuta, S., Sato, K., Kashiwadani, H., Tsunoda, K., Yamasoba, T., and Mori, K. (2010). From the Cover: neurons in the anterior olfactory nucleus pars externa detect right or left localization of odor sources. Proc. Natl. Acad. Sci. U.S.A. 107, 12363-12368. doi: 10.1073/pnas.1003999107

Koehl, M. A., Koseff, J. R., Crimaldi, J. P., McCay, M. G., Cooper, T., Wiley, M. B., et al. (2001). Lobster sniffing: antennule design and hydrodynamic filtering of information in an odor plume. Science 294, 1948-1951. doi: 10.1126/science. 1063724

Kuenen, L. P. S., and Carde, R. T. (1994). Strategies for recontacting a lost pheromone plume - casting and upwind flight in the male gypsy-moth. Physiol. Entomol. 19, 15-29. doi: 10.1111/j.1365-3032.1994.tb01069.x

Liu, A., Papale, A. E., Hengenius, J., Patel, K., Ermentroud, B., and Urban, N. N. (2020). Mouse navigation strategies for odor source localization. bioRxiv [Preprint]. doi: 10.1101/558643

Manurung, A. (2016). Numerical Methods For Velocity Estimation. Available at: https://www.mathworks.com/matlabcentral/fileexchange/52381-numericalmethods-for-velocity-estimation (accessed March 3, 2020).

Marsh, D., Kennedy, J. S., and Ludlow, A. R. (1978). An analysis of anemotactic zigzagging flight in male moths stimulated by pheromone. Physiol. Entomol. 3, 221-240. doi: 10.1111/j.1365-3032.1978.tb00152.x

Martin, H. (1965). Osmotropotaxis in Honey-Bee. Nature 208:59. doi: 10.1038/ 208059a0

Means, L. W., Alexander, S. R., and Oneal, M. F. (1992). Those cheating rats - male and female rats use odor trails in a water-escape working memory task. Behav. Neural Biol. 144-151. doi: 10.1016/0163-1047(92)90387-J

Papale, A. E., Stott, J. J., Powell, N. J., Regier, P. S., and Redish, A. D. (2012). Interactions between deliberation and delay-discounting in rats. Cogn. Affect. Behav. Neurosci. 12, 513-526. doi: 10.3758/s13415-012-0097-7

Pierce-Shimomura, J. T., Morse, T. M., and Lockery, S. R. (1999). The fundamental role of pirouettes in Caenorhabditis elegans chemotaxis. J. Neurosci. 19, 9557-9569.
Porter, J., Craven, B., Khan, R. M., Chang, S. J., Kang, I., Judkewitz, B., et al. (2007). Mechanisms of scent-tracking in humans. Nat. Neurosci. 10, 27-29. doi: $10.1038 / \mathrm{nn} 1819$

Schwenk, K. (1994). Why snakes have forked tongues. Science 263, 1573-1577. doi: $10.1126 /$ science.263.5153.1573

Steck, K., Knaden, M., and Hansson, B. S. (2010). Do desert ants smell the scenery in stereo? Anim. Behav. 79, 939-945. doi: 10.1016/j.anbehav.2010. 01.011

Sutton, R. S., and Barto, A. G. (1998). Reinforcement Learning: An Introduction. Cambridge, Mass: MIT Press.

Tao, L. Y., Ozarkar, S., Beck, J. M., and Bhandawat, V. (2019). Statistical structure of locomotion and its modulation by odors. eLife 8:41235. doi: 10.7554/eLife. 41235

Thesen, A., Steen, J. B., and Doving, K. B. (1993). Behavior of dogs during olfactory tracking. J. Exp. Biol. 180, 247-251.

Vergassola, M., Villermaux, E., and Shraiman, B. I. (2007). 'Infotaxis' as a strategy for searching without gradients. Nature 445, 406-409. doi: 10.1038/ nature 05464

Vickers, N. J. (2000). Mechanisms of animal navigation in odor plumes. Biol. Bull. 198, 203-212. doi: 10.2307/1542524

Wallace, D. G., Gorny, B., and Whishaw, I. Q. (2002). Rats can track odors, other rats, and themselves: implications for the study of spatial behavior. Behav. Brain Res. 131, 185-192. doi: 10.1016/S0166-4328(01) 00384-9

Wesson, D. W., Donahou, T. N., Johnson, M. O., and Wachowiak, M. (2008). Sniffing behavior of mice during performance in odor-guided tasks. Chem. Sens. 33, 581-596. doi: 10.1093/chemse/bjn029

Willis, M. A., Avondet, J. L., and Finnell, A. S. (2008). Effects of altering flow and odor information on plume tracking behavior in walking cockroaches, Periplaneta americana (L.). J. Exp. Biol. 211(Pt 14), 2317-2326. doi: 10.1242/ jeb.016006

Wilson, D. A., and Sullivan, R. M. (1999). Respiratory airflow pattern at the rat's snout and an hypothesis regarding its role in olfaction. Physiol. Behav. 66, 41-44. doi: 10.1016/S0031-9384(98)00269-8

Zhang, S. J., and Manahan-Vaughan, D. (2015). Spatial olfactory learning contributes to place field formation in the hippocampus. Cereb. Cortex 25, 423-432. doi: 10.1093/cercor/bht239

Zhao, W. Q., Gong, C. X., Ouyang, Z. H., Wang, P. F., Wang, J., Zhou, P. P., et al. (2017). Turns with multiple and single head cast mediate drosophila larval light avoidance. PLoS One 12:e0181193. doi: 10.1371/journal.pone.0181193

Conflict of Interest: The authors declare that the research was conducted in the absence of any commercial or financial relationships that could be construed as a potential conflict of interest.

Copyright (c) 2020 Liu, Papale, Hengenius, Patel, Ermentrout and Urban. This is an open-access article distributed under the terms of the Creative Commons Attribution License (CC BY). The use, distribution or reproduction in other forums is permitted, provided the original author(s) and the copyright owner(s) are credited and that the original publication in this journal is cited, in accordance with accepted academic practice. No use, distribution or reproduction is permitted which does not comply with these terms. 